\title{
Quantifying Spatiotemporal Dynamics of Vegetation and Its Differentiation Mechanism Based on Geographical Detector
}

\section{Guangjie Wang}

Sichuan Normal University

wenfu peng ( $\sim$ pwfzh@126.com )

Sichuan Normal University

\section{Research Article}

Keywords: Normalized vegetation index (NDVI), impact factors, Geographical Detector, Geographic information system (GIS), Minjiang River

Posted Date: September 3rd, 2021

DOl: https://doi.org/10.21203/rs.3.rs-723483/v1

License: (c) (i) This work is licensed under a Creative Commons Attribution 4.0 International License.

Read Full License

Version of Record: A version of this preprint was published at Environmental Science and Pollution Research on January 11th, 2022. See the published version at https://doi.org/10.1007/s11356-02117544-w. 
2 1.Funding for this study was provided by the Humanities and Social Science Research Foundation 3 of Ministry of Education, China (No.17YJA850007) and National Natural Science Foundation of

4 China (No.41371125). The funding sources had no involvement in the collection, analysis and

5 interpretation of data; the writing of the report; and the decision to submit the article for

6 publication.

7 2.This manuscript has not been published or presented elsewhere in part or in entirety, and is not

8 under consideration by another journal. All of the authors contributed materially to the study, have

9 reviewed and approved the manuscript, and agreed with submission to the journal. The authors

10 have no conflicts of interest to declare.

11 All of the authors:

12 Guangjie Wang,Wenfu Peng

\section{Consent to Participate}

All of the authors contributed materially to the study, have reviewed and approved the manuscript, and agreed with submission to the journal. The authors have no conflicts of interest to 17 declare.

All of the authors:

Guangjie Wang,Wenfu Peng

The Institute of Geography and Resources, Sichuan Normal University, Chengdu 610068, P. R. China

\section{Consent to Publish}

This manuscript has not been published or presented elsewhere in part or in entirety, and is not under consideration by another journal. All of the authors contributed materially to the study, have reviewed and approved the manuscript, and agreed with submission to the journal. The authors have no conflicts of interest to declare.

27 All of the authors: 
32

1.Guangjie Wang: Quantifying spatiotemporal dynamics of vegetation

2.Quantifying the mechanisms of patiotemporal dynamics of vegetation based on geographical detector

All of the authors:

Guangjie Wang,Wenfu Peng

The Institute of Geography and Resources, Sichuan Normal University, Chengdu 610068, P. R. China

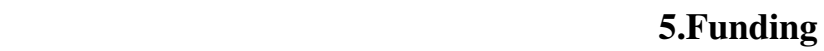

This manuscript has not been published or presented elsewhere in part or in entirety, and is not under consideration by another journal. All of the authors contributed materially to the study, have reviewed and approved the manuscript, and agreed with submission to the journal. The authors have no conflicts of interest to declare.

All of the authors:

Guangjie Wang,Wenfu Peng

1. The Institute of Geography and Resources Science, Sichuan Normal University, Chengdu 610068, China; 
All of the authors have reviewed and approved the manuscript, and agreed with availability of data and materials submission to the journal. The authors have no conflicts of interest to declare.

64 All of the authors:

80

81

82

83

84

85

86

87

88

89

90 


\section{Quantifying spatiotemporal dynamics of vegetation and its differentiation mechanism based on geographical detector}

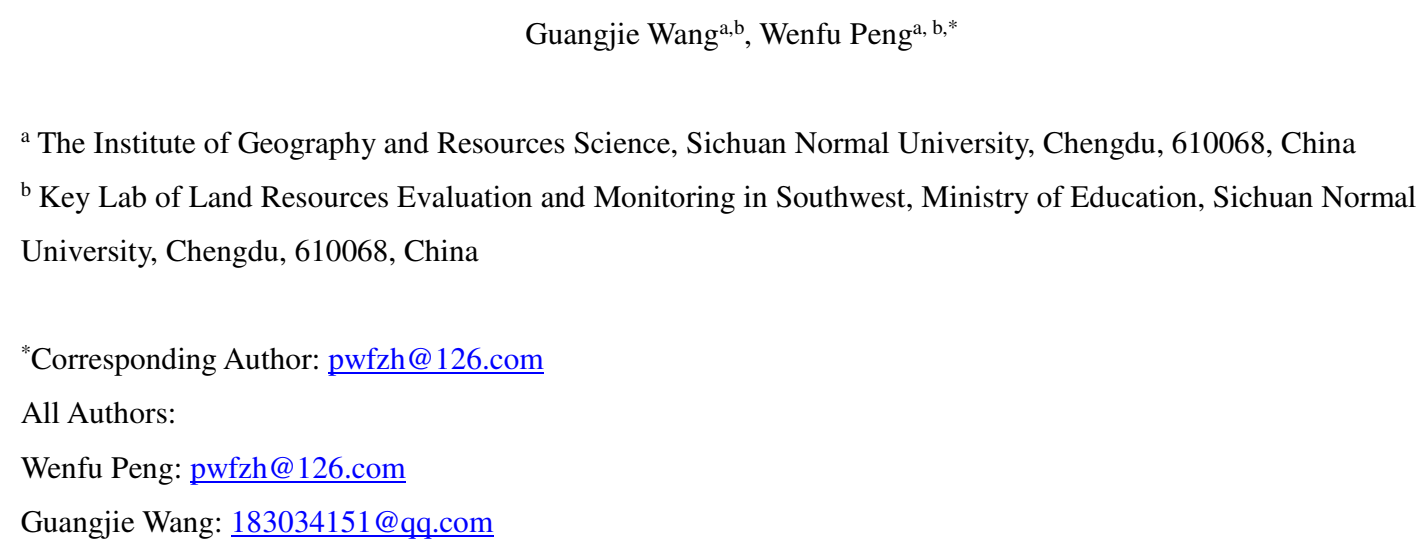

Abstract: Understanding of the influence of factors on vegetation changes in different is still largely unknown. We have applied the Geographic Detector, a new spatial statistical method to study the interactive effects of factors on the spatial patterns of normalized vegetation index (NDVI) changes and determine the optimal characteristics of key impact factors that are beneficial to vegetation growth. Our results show that the vegetation cover from 2000 to 2015 for the upper reaches of the Minjiang River, western China was in good condition, the areas of $0.6<\mathrm{NDVI}>0.8$ and NDVI $>0.8$ accounted for more than $80 \%$, and the spatial-temporal changes of vegetation cover were significant. The changes of vegetation cover were showed a significant transformation in the regions of NDVI>0.6. Our study uniquely illustrated that the elevation, annual average temperature and soil type can relatively well explain the vegetation changes. We propose that there are interactive effects between impact factors on vegetation NDVI, and the synergistic effects of the impact factors show mutual enhancement and nonlinear enhancement. The interactions between impact factors significantly enhance the impact of a single factor on vegetation changes. The most suitable characteristics of the main impact factors that promote the vegetation growth revealed help a better understanding of the impact of factors on NDVI and its driving mechanisms. Our findings suggest that the determined a favourable value range or the most suitable characteristics of impact factors help to intervene and promote vegetation change for vegetation restoration and alleviate environmental degradation.

Key words: Normalized vegetation index (NDVI), impact factors, Geographical Detector, Geographic information system (GIS), Minjiang River

\section{Introduction}


Surface vegetation not only is the main body of terrestrial ecological environment, but also plays a leading role in maintaining the functions of terrestrial ecosystem (Liu et al.,2019). Moreover, vegetation is a sensitive and vulnerable factor reflecting the changes of ecological environment. Vegetation cover is an important remote sensing indicator for ecosystem monitoring and evaluation of surface processes, and also an important variable for understanding ecosystem processes, the health condition of vegetation and the carbon storage (Luke Wallace et al.,2019; Yinghai Ke et al.,2016). Therefore, the study of vegetation changes and their driving factors at regional and global scales is of great significance for monitoring the evolution of terrestrial ecosystem and change in ecological environment, managing natural resources, and developing strategies to adapt to environmental changes (Liu et al.,2013; Peng et al.,2019; Liu et al.,2019).

With the development of earth observation technologies, the vegetation indexes based on remote sensing are often used for vegetation dynamic monitoring (Peng et al.,2019).The normalized vegetation index (NDVI) has been widely used in the research of spatial-temporal scale changes of vegetation. At present, domestic and foreign scholars have applied remote sensing data and different research methods to study the vegetation changes and their driving mechanisms. C.Piedallu et al. (2019) calculated spring greenness and annual relative greenness to improve understanding of the different environmental drivers of NDVI spatial variations for different stand type characteristics of mountain and Mediterranean biomes, and concluded that the NDVI dynamics was not only driven by climatic variability, the different environmental factors act complementarily and soil parameters characterizing water stress and soil nutrition should be taken into account. Based on abiotic and biotic datasets from 735 quadrats of natural range lands located in the southern Alborz Province of Iran,the topographic variables, grazing disturbance intensities, soil textural properties as predictor variables were quantified, found that vegetation quantity and quality were jointly explained by topography, grazing disturbances, and soil textural properties (Anvar Sanaei et al.,2019). The vegetation cover in a typical semi-humid and semi-arid region under changing environments, the Wei River Basin, and its reasons were studied by investigating the relationships among precipitation, temperature and soil moisture condition using the cross wavelet technique, showed that vegetation cover is more influenced by temperature and soil moisture than precipitation (Liu et al.,2019a). Mo et al.(2019) used remote sensing data of precipitation and NDVI to investigate the correlation at different temporal and spatial scales in a 
typical arid mountain-oasis river system located in northwest China, and found that strong effects of precipitation on NDVI in the study area, the correlation coefficient between precipitation and NDVI varied spatially with the precipitation pattern in space. Chen et al. (2019) analyzed vegetation cover and its correlation with climate factors in Qinba mountains by using the methods of linear trend analysis and related analysis, and found that vegetation cover and temperature are mainly positively correlated, and coexists with precipitation positively and negatively,the correlation with temperature is higher than that of precipitation. Ma et al. (2018) combined correlation analysis, residual trend analysis and other methods, the change of grassland vegetation cover of the growing season in Qinghai province and the influence of climate factors and human activities were analyzed, and showed that enhanced vegetation index in growing season meadow is significantly correlated with temperature and precipitation,and more closely correlated with temperature. Yao et al.(2019) studied the spatial heterogeneity of vegetation greenness and its relationship with vegetation greening in China from 2000 to 2017, using MODIS enhanced vegetation index, and suggested that vegetation greening may increase the heterogeneity of vegetation greenness space and have a series of effects on the environment and human activities. Based on NDVI, terrain, precipitation and temperature data, the vegetation growth and driving mechanism in the Luzangbo River Basin from 1982 to 2010 were analyzed, and found that the relationship between vegetation growth and climate changed significantly with the increase of drought degree, NDVI in arid areas is positively correlated with temperature, while that in semi-arid areas is negatively correlated with temperature (Sun et al.,2019). Liu et al. (2013) studied the fractional vegetation cover (FVC) change trend and its relationship with climate change on the Tibet Plateau from 1981 to 2005, and concluded that the vegetation coverage increases as a whole and degenerates as precipitation increases, FVC change is positively correlated with precipitation change and temperature change in the same period, and there is a regional difference. Ma et al.(2019) presented a pollen record covering the last deglaciation and Holocene from Lake Tangra Yumco on the Tibet Plateau, inferred a regional vegetation and climate history during the late glacial period, it is found that vegetation and climate change in the central region of Tibet Plateau, are mainly driven by sunshine, but it is also related to the impact of sudden cold events in the north Atlantic region during the holocene. Zhu et al. (2019) analyzed the spatiotemporal change and its driving forces of vegetation coverage in the upper reaches of 
Minjiang River from 2006 to 2016, using unitary linear regression analysis, stability analysis method and geographic detector model, it is considered that vegetation coverage is mainly affected by elevation, temperature, soil type, precipitation and other factors.Yang et al. (2018) analyzed the spatial patterns of FVC and its variation rules and its response to climate factors in Zoige area from 2000 to 2015, based on the methods of trend analysis, correlation analysis and hysteresis analysis,it is concluded that the vegetation coverage in the growing season has a good correlation with the temperature in the same period, and it has obvious hysteresis to precipitation, most areas are affected by non-temperature and precipitation factors. Xiong et al. (2018) studied the multi-scale coupling relationship between NDVI and environmental factors such as elevation, slope, slope direction, distance from the residential area and distance from river in Maoxian of the upper Minjiang River, based on the two-dimensional wavelet transform and Landsat 8 OLI remote sensing images and DEM data in 2015, and the spatial distribution of NDVI was determined by environmental factors. The correlation between vegetation coverage and topographic factors in the upper reaches of Minjiang River was analyzed quantitatively by means of spatial superposition analysis and construction of statistical samples,using Landsat 8 OLI remote sensing image and DEM data to extract vegetation coverage and terrain factors, and suggested that the vegetation coverage of the upper Minjiang River basin is closely related to the topographic factors and the variation of topographic factors has an important impact on the ecological environment (Zhang et al., (2018).

However, most of the above studies focus on the impact of climatic factors or natural factors on vegetation cover changes and establish the relationship between vegetation cover changes and climatic factors or natural factors to explore and describe the changes and characteristics of vegetation cover. Although the findings are important and have greatly promoted the understanding of vegetation changes and their driving mechanisms, most of the methods involved in the studies apply linear analysis, trend analysis and correlation analysis. The research assumes that there is a significant linear relationship between the vegetation changes and their driving forces, which is not consistent with the actual situation (Peng et al.,2019). At the same time, with the development and popularization of positioning and observation technologies, in either the more elaborate or larger research or spatial big data, the problem of spatial heterogeneity is highlighted, and the statistical methods aimed for spatial heterogeneity are still very limited (Wang 
et al.,2017). Although hundreds of classification algorithms such as k-means and SOM have been used for classification or partitioning, statistical methods for spatial differentiation are still very limited (Wang et al.,2010; Wang et al., 2016). Currently, the main methods include Geographic Detector models for spatial heterogeneity measurement and factor analysis (Wang et al., 2016). Geographic detector is a new tool for measuring, mining and utilizing spatial heterogeneity, which theoretical core is to detect the consistency of spatial distribution pattern between dependent variable and independent variable through spatial heterogeneity, and to measure the degree of explanation of independent variable to dependent variable (Wang et al.,2017). The Geographic Detector model has a stronger power than the general statistics, is more confident, and strongly suggests a causal relationship, because it is much more difficult for two variables to be uniformly distributed in two dimensions than for two variables to be uniformly distributed in one dimension.

The improvement of vegetation coverage in the upper reaches of the Minjiang River is of great significance for the construction of ecological barriers in the upper reaches of the Yangtze River basin, supporting and serving regional and national survival and development, ecological civilization construction, rural revitalization, etc. At present, how the changes of various impact factors and their superposition effects influence the vegetation cover changes receives less attention (Peng et al.,2019). It is nevertheless difficult to quantitatively analyse factors influencing differences in NDVI variation (Wang et al., 2016). Identifying the causes of vegetation cover changes is still challenging, yet this is important for helping us to understand the connections between the multi-factor in the plateau environment for the upper reaches of the Minjiang River and the changes in vegetation cover. However, the existing studies rarely discuss the relationship between vegetation cover changes and their multi-factor. In addition to climatic factors, other natural factors (such as elevation, slope, aspect, soil, vegetation, etc) and humanity factors (GDP density, population density, distance from roads or rivers or town centers, etc.) also have relatively great impact on vegetation cover changes. Compared with the role of single factor, the changes of vegetation cover are more affected by the variations of multiple impact factors, especially in the upper reaches of the Minjiang River where the topography is complex and multi-factor has complicated effects on vegetation cover changes.

The objectives in this paper are as follows: 1) identify the main impact factors and their role in vegetation cover change; 2) distinguish whether the impact factors for the upper reaches of the 
Minjiang River in Sichuan province are independent or interdependent influences on vegetation

change; and 3) determine the optimum characteristics of the impact factors most conducive to vegetation growth.This paper consists of five parts: the first section is the introduction; the second section introduces the research area, data sources and research methods; the third section reports the main results and findings of vegetation cover change and influences on factors on NDVI change; the fourth section give all discussion on impact factors within the optimum characteristics or value range serve to promote vegetation changes; the fifth section summarizes the main results of quantifying influences of impact factors.

\section{Data sources and methods}

\subsection{Study area}

The upper reaches of the Minjiang River, western China is located on the eastern edge of the Qinghai-Tibet Plateau and sited in the transition zone between the hilly lands surrounding the Sichuan Basin and the Qinghai-Tibet Plateau, within the region of $102^{\circ} 34^{\prime} \sim 104^{\circ} 14^{\prime} \mathrm{E}$, $30^{\circ} 45^{\prime}-33^{\circ} 12^{\prime} \mathrm{N}$, which includes Songpan, Heishui, Mao County, Li County and Wenchuan, covering an area of $24,753.42 \mathrm{~km}^{2}$. The geomorphic type of the upper reaches of the Minjiang River is dominated by plateaus and alpine valleys. The elevation rises from $762 \mathrm{~m}$ in the southeast to $5870 \mathrm{~m}$ in the northwest. The terrain is undulating, being high in the northwest and low in the southeast, with an average elevation of about $3400 \mathrm{~m}$. The geological structure is complex, and the new tectonic activities are intense, bringing many natural disasters such as earthquakes, landslides and debris flows. The climatic division of the upper reaches of the Minjiang River includes the mid-subtropical, northern subtropical and plateau climate zones. The climate varies from subtropical to temperate, frigid temperate and frigid, with obvious vertical differences. It has the characteristics of distinct dry and rainy seasons and uneven distribution of precipitation. The annual average precipitation is about $637.7 \mathrm{~mm}$, and more than $80 \%$ of the rainfall is concentrated in May to October. The rainfall is mostly strong and short-lived heavy rain. The temperature is relatively low, with small annual difference but large daily difference. The annual average temperature is $11^{\circ} \mathrm{C}$. The sunshine is sufficient, and the annual average land surface evaporation is $793.4 \mathrm{~mm}$. The foehn effect in the valleys is significant thereby the annual rainfall there is less than $500 \mathrm{~mm}$ while the evaporation is $1340 \mathrm{~mm}$. With the changes in elevation and hydrothermal conditions, vegetation and soil types show remarkable vertical zonality. Vegetation types include 
forests, subalpine coniferous forests, subalpine meadows, alpine shrubs, arid valley shrubs and

other ecological types. Soil types include cinnamon soil, brown soil, dark brown soil, subalpine meadow soil, etc., all of which have typical vertical structures. In 2015, the population of the upper reaches of the Minjiang River was $39.17 \times 10^{4}$ people, and the total GDP was $148.73 \times 10^{8}$ yuan, in which the proportions of the first, second and third industries were $10.11 \%, 64.77 \%$ and $25.12 \%$, respectively.

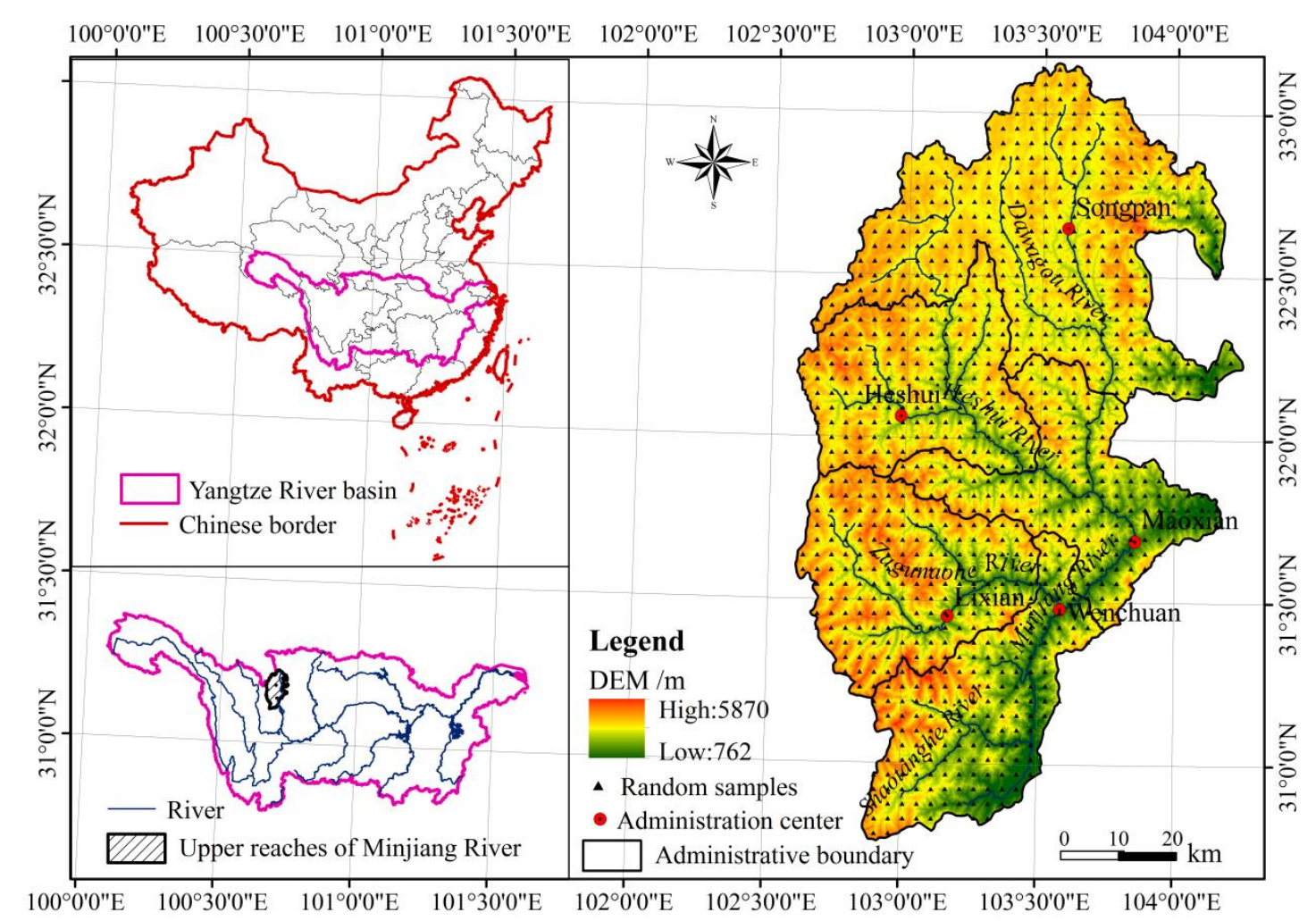
factors,climate, geomorphy, topography,vegetation and soil (Tab.1). NDVI and DEM are derived climate, topography, vegetation, soil and Landsat 8 images are all from the Data Center of 291 Resources and Environmental Sciences of the Chinese Academy of Sciences(http://www.resdc.cn). The land use data was derived from the interpretation of the Landsat 8 remote sensing image. The

\subsection{Data sources}

Fig.1. Location of study area

This study data in this paper included NDVI, Digital Elevation Model (DEM), humanity from the United States Geological Survey (http://gdex.cr.usgs.gov/gdex/). NDVI data was calculated by MODIS MOD13Q1 products, and the sinusoidal projection of the MODIS MOD 13Q1 product was converted into a UTM projection and the coordinate system of the projection is set to WGS_84 based on the MODIS re-projection tool (MRT). GDP density, population density, 
distance from county, township, road or river was obtained by GIS. GDP density and population density were calculated by applying the multi-factor weight distribution method, the GDP data or population with administrative region as the basic statistical unit is distributed to the grid unit based on land use type, night light brightness, residential density and other factors closely related to human economic activities, respectively. Terrain data, i.e. elevation, aspect and slope were derived from the DEM. Climate map was extracted the interpolation of inverse distance weighted average method and DEM correction based on 1915 meteorological stations in China. Climate zoning data were compiled by the national meteorological administration in 1978, by using climatic data from 1951 to 1970 . Soil map was compiled and published by the Chinese Soil Census Office in 1995. The vegetation map was digitized by 1:10 000 vegetation map. The research data was uniformly set to the WGS_1984 projection coordinates and the GCS_WGS_1984 geographic coordinates.

Tab. 1 The factors indicators in study area

\begin{tabular}{|c|c|c|c|c|}
\hline Category & Code & Index & Unit & Resolution \\
\hline \multirow[t]{7}{*}{ Humanity } & $x_{1}$ & GDP density & $\mathrm{RMB}$ yuan $/ \mathrm{km}^{2}$ & $1 \mathrm{~km}$ \\
\hline & $x_{2}$ & Population density & person $/ \mathrm{km}^{2}$ & $1 \mathrm{~km}$ \\
\hline & $x_{3}$ & Land use & class & $1 \mathrm{~km}$ \\
\hline & $x_{4}$ & Distance from county seat & $\mathrm{m}$ & $30 \mathrm{~m}$ \\
\hline & $x_{5}$ & Distance from town & $\mathrm{m}$ & $30 \mathrm{~m}$ \\
\hline & $x_{6}$ & Distance from road & $\mathrm{m}$ & $30 \mathrm{~m}$ \\
\hline & $x_{7}$ & Distance from river & $\mathrm{m}$ & $30 \mathrm{~m}$ \\
\hline \multirow[t]{6}{*}{ Climate } & $x_{8}$ & Annual average temperature & ${ }^{\circ} \mathrm{C}$ & $1 \mathrm{~km}$ \\
\hline & $x_{9}$ & Cumulative temperature $\left(\geq 10^{\circ} \mathrm{C}\right)$ & ${ }^{\circ} \mathrm{C}$ & $1 \mathrm{~km}$ \\
\hline & $x_{10}$ & Average annual precipitation & $\mathrm{mm}$ & $1 \mathrm{~km}$ \\
\hline & $x_{11}$ & Humidity index & level & $1 \mathrm{~km}$ \\
\hline & $x_{12}$ & Dryness index & level & $1 \mathrm{~km}$ \\
\hline & $x_{13}$ & Global radiation & $\mathrm{MJ} / \mathrm{m}^{2}$ & $1 \mathrm{~km}$ \\
\hline Geomorphy & $x_{14}$ & Types & class & $1 \mathrm{~km}$ \\
\hline \multirow[t]{3}{*}{ Topography } & $x_{15}$ & Elevation & $\mathrm{m}$ & $30 \mathrm{~m}$ \\
\hline & $x_{16}$ & Slope & $\circ$ & $30 \mathrm{~m}$ \\
\hline & $x_{17}$ & Aspect & $\circ$ & $30 \mathrm{~m}$ \\
\hline Vegetation & $x_{18}$ & Vegetation types & types & $1 \mathrm{~km}$ \\
\hline Soil & $x_{19}$ & Soil types & types & $1 \mathrm{~km}$ \\
\hline
\end{tabular}


(1) Synthesis method of NDVI: the upper reaches of Minjiang River is not only an area

integrating multiple areas such as ecological barrier area, biodiversity area, Plateau Tibetan area, typical fragile ecological environment area and relatively concentrated poor population, but also a complex system integrating irreplaceable functions such as resource supply, ecological services and environmental regulation with economic and social development. To describe the characteristics of interannual NDVI more comprehensively, the Savitzky-Golay filter in this study is used to reconstruct the MODIS NDVI data to eliminate the influence of noise; the annual maximum NDVI is synthesized by the maximum value composite (MVC) method, which effectively reduces the influence of factors such as cloud, atmosphere and solar elevation angle, and the vegetation NDVI data of the year 2000, 2005, 2010 and 2015 is synthesized.

(2) Levels: in order to better analyse the dynamic change of vegetation cover, based on the calculation of vegetation NDVI from 2000 to 2015, according to the NDVI and combined with the actual situation of the upper reaches of the Minjiang River (Peng et al., 2016), the vegetation NDVI is divided into 5 vegetation cover levels using the equal spacing method: low vegetation cover $(0 \sim 0.2]$, mid-low vegetation cover $(0.2 \sim 0.4]$, middle vegetation cover $(0.4 \sim 0.6]$, mid-high vegetation cover (0.6 0.8] and high vegetation cover (0.8 1.0] (peng et al.,2019). Microsoft Excel was used for data analysis.

\subsubsection{Image density segmentation and difference image algorithm}

The setting of vegetation coverage threshold will affect the calculation of vegetation coverage area. Considering the actual vegetation coverage for the upper reaches of Minjiang River, field survey data, if the thresholds of vegetation coverage is set to $10 \%$, regions with a decrease in vegetation coverage of $0 \sim 10 \%$ may be omitted (Peng et al., 2016), dynamic changes in vegetation coverage were extracted using image density segmentation and image differencing algorithms. Vegetation coverage is considered to remain unchanged, decrease, or increase if the difference values of vegetation coverage are zero, negative, or positive respectively (Peng et al., 2019).

\subsubsection{Index selection and information extraction}

(1) Index selection: the mountain system of the upper Minjiang is an extension on the Tibet Plateau,the formation of geomorphic types is complex, the diversity of climate, vegetation and soil and the significant vertical gradient, and the strong disturbance of human activities lead to the complexity and fragility of the ecological environment. According to the index system of selection 
of systematic, typicality, dynamic, scientific, quantifiable, and can obtain the principle, only 6 categories and 19 factors including humanity factor, climate, geomorphic type, terrain, vegetation and soil data were selected to explore the influence of factors on vegetation changes in the upper Minjiang River (Tab.1).

(2) Information extraction: some 24709 randomly sampling points files were generated, invalid samples were deleted, and 23,161 valid samples were obtained based on $1 \mathrm{~km} \times 1 \mathrm{~km}$ grids by using a GIS (Fig.1).Then, according to the spatial position, the NDVI of sampling points was correlated with all factor data, thus generating an attribute table, and calculate the quantitative relationship between the corresponding NDVI and the selection of each index (Peng et al.,2019).

\subsubsection{Grading of impact factors}

Land use, geomorphic type, soil and vegetation were each classified into $6,7,18$ and 8 classes according to their types, respectively. According to the natural break point method (Liu et al., 2017), the GDP density, population density, distance from county, distance from town, distance from road, distance from river, annual average temperature, accumulated temperature $\left(\geq 10^{\circ} \mathrm{C}\right)$, annual average precipitation, wetness index, total radiation, elevation and slope were each divided into 12 classes, aspect into 9 classes, and dryness index into 6 classes, respectively (Tab.1).

\subsubsection{Variable coefficient}

The fluctuation of NDVI in the upper reaches of Minjiang River from 2000 to 2015 was calculated by the variable coefficient, and the formula is as follows:

$$
C_{v}=\frac{1}{\bar{F}} \sqrt{\frac{\sum_{i=1}^{n}\left(F_{i}-\bar{F}\right)^{2}}{n-1}} \times 100 \%
$$

Where the $C_{v}$ is variable coefficient, $n$ is monitoring the number of years, $\bar{F}$ is mean NDVI in study phase, $F_{i}$ is NDVI in $i$ year.

\subsubsection{Geographical Detector model}

Geographical Detectors model represent a new spatial statistics method that is used to detect spatial heterogeneity and identify driving factors based on risk, factors, ecology, and interaction (Wang et al., 2017; Wang et al.,2010). By calculating and comparing the $q$ value of each single 
factor and the $q$ value after the superposition of two factors, the Geographic Detector model can judge whether there is interaction between the two factors and whether the interaction is strong or weak, square, linear or nonlinear.

(1) Detection of spatial heterogeneity and factors. The calculation method comprises the following steps: Firstly, spatial overlay analysis was performed for the NDVI layer and factor layer; secondly, factors were divided into different spatial types or subzones; and thirdly, a significance test for the differences of mean values of factors was conducted, to detect relative importance of factors. The calculation model of the explanatory power of each factor is as follows:

$$
q=1-\frac{\sum_{h=1}^{L} N_{h} \sigma_{h}^{2}}{N \sigma^{2}}=1-\frac{S S W}{S S T}
$$

Where $q$ is the explanatory power of factors on vegetation $N D V I, h=1, \cdots, L$ are the stratification of $y$ or factor $x$, that is, classification or partition; $N_{h}$ and $N$ are the number of units in $h$ and the whole region,respectively. $N$ and $\sigma^{2}$ are the total number of samples and the variance of y value in the whole region. $N_{h}$ is the variance of units $h$.

The range of $q$ value is $[0,1]$, and the larger the $q$ value is, the more obvious the spatial differentiation of $y$ is. In the extreme case, the $q$ value of 1 indicates that factor $x$ completely controls the spatial distribution of $Y$, the $q$ value of 0 indicates that the factor $x$ has nothing to do with $Y$.

The variance calculation formula of the y value in the whole region is as follows:

$$
\sigma^{2}=\frac{1}{N-1} \sum_{i=1}^{N}\left(Y_{i}-\bar{Y}\right)^{2}
$$

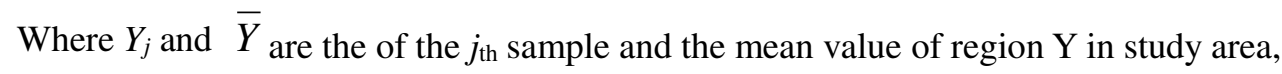
respectively.

$$
\sigma_{h}^{2}=\frac{1}{N_{h}-1} \sum_{i=1}^{N_{h}}\left(Y_{h, i}-\bar{Y}_{h}\right)^{2}
$$

where $Y_{h, i}$ and $\bar{Y}$ is the value of $i$ th sample and the mean of $Y$ in zone $h$, respectively.

(2) Detection of factor interaction. Interaction detection is used to identify the interaction between factors, that is, to evaluate the accountability of the combined effect (enhancing or weakening) and 
respective effect on the NDVI. First, the $q$ values of two factors with respect to NDVI were calculated $\left(q\left(x_{i}\right)\right.$ and $q\left(x_{j}\right)$. Then, $q$ values regarding the interaction between factors was calculated $\left(q\left(x_{i} \cap x_{j}\right)\right)$ and compared with $q\left(x_{i}\right)$ and $q\left(x_{j}\right)$.

(3) Detection of risk zones. Risk detection is used to judge whether there is a significant difference in mean attribute values between the subzones of two factors, and can be used to find regions with high vegetation coverage. The risk detection is examined by using $t$ statistic value:

$$
t=\frac{\bar{Y}_{h=i}-\bar{Y}_{h=j}}{\left[\frac{\operatorname{Var}\left(Y_{h=i}\right)}{n_{h}=1}+\frac{\operatorname{Var}\left(Y_{h=j}\right)}{n_{h}=2}\right]^{1 / 2}}
$$

\section{Results and analysis}

\subsection{Dynamic change in NDVI}

The regions with middle $(0.6<\mathrm{NDVI}<0.8)$ and high vegetation cover $(0.8<\mathrm{NDVI}<1)$ areas accounted for $17 \%$ and $68 \%$ in 2000 and $20 \%$ and $65 \%$ or higher in 2015 of the upper reaches of the Minjiang River, respectively. The region with low $(0<\mathrm{NDVI}<0.2)$, mid-low $(0.2<\mathrm{NDVI}<0.4)$, or middle vegetation cover $(0.4<\mathrm{NDVI}<0.6)$ area only accounted for less than $14 \%$ in 2000 and 2015 of the upper reaches of the Minjiang River (Tab.2). This shows that the vegetation cover in the study area is extensive. However, the regions with $0.8<\mathrm{NDVI}<1$ were reduced. From 2000 to 2015 , the regions with $0<\mathrm{NDVI}<0.2,0.2<\mathrm{NDVI}<0.4,0.4<\mathrm{NDVI}<0.6$, and $0.6<\mathrm{NDVI}<0.8$ showed a rising trend, and the increases in their proportions in the study area were $0.03 \%, 0.13 \%, 0.49 \%$, and $2.94 \%$, respectively. The regions with $0.8<\mathrm{NDVI}<1$ showed a relatively large decreasing trend, with a reduction rate of $3.60 \%$.

Tab.2 Dynamic changes of NDVI during 2000 2015

\begin{tabular}{lcccccc}
\hline Year & \multicolumn{2}{c}{2000} & \multicolumn{2}{c}{2015} & \multicolumn{2}{c}{$2000 \sim 2015$} \\
\hline NDVI class & Area $/ \mathrm{km}^{2}$ & Proportion $/ \%$ & Area $/ \mathrm{km}^{2}$ & Proportion /\% & Area change $/ \mathrm{km}^{2}$ & Proportion /\% \\
\hline$(0 \sim 0.2]$ & 1111.50 & 4.49 & 1118.06 & 4.52 & 6.56 & 0.03 \\
$(0.2 \sim 0.4]$ & 828.50 & 3.35 & 861.69 & 3.48 & 33.19 & 0.13 \\
$(0.4 \sim 0.6]$ & 1388.25 & 5.61 & 1510.25 & 6.11 & 122.00 & 0.49 \\
$(0.6 \sim 0.8]$ & 4341.69 & 17.55 & 5069.50 & 20.49 & 727.81 & 2.94 \\
$(0.8 \sim 1.0]$ & 17066.50 & 68.99 & 16176.94 & 65.40 & -889.56 & -3.60 \\
\hline
\end{tabular}



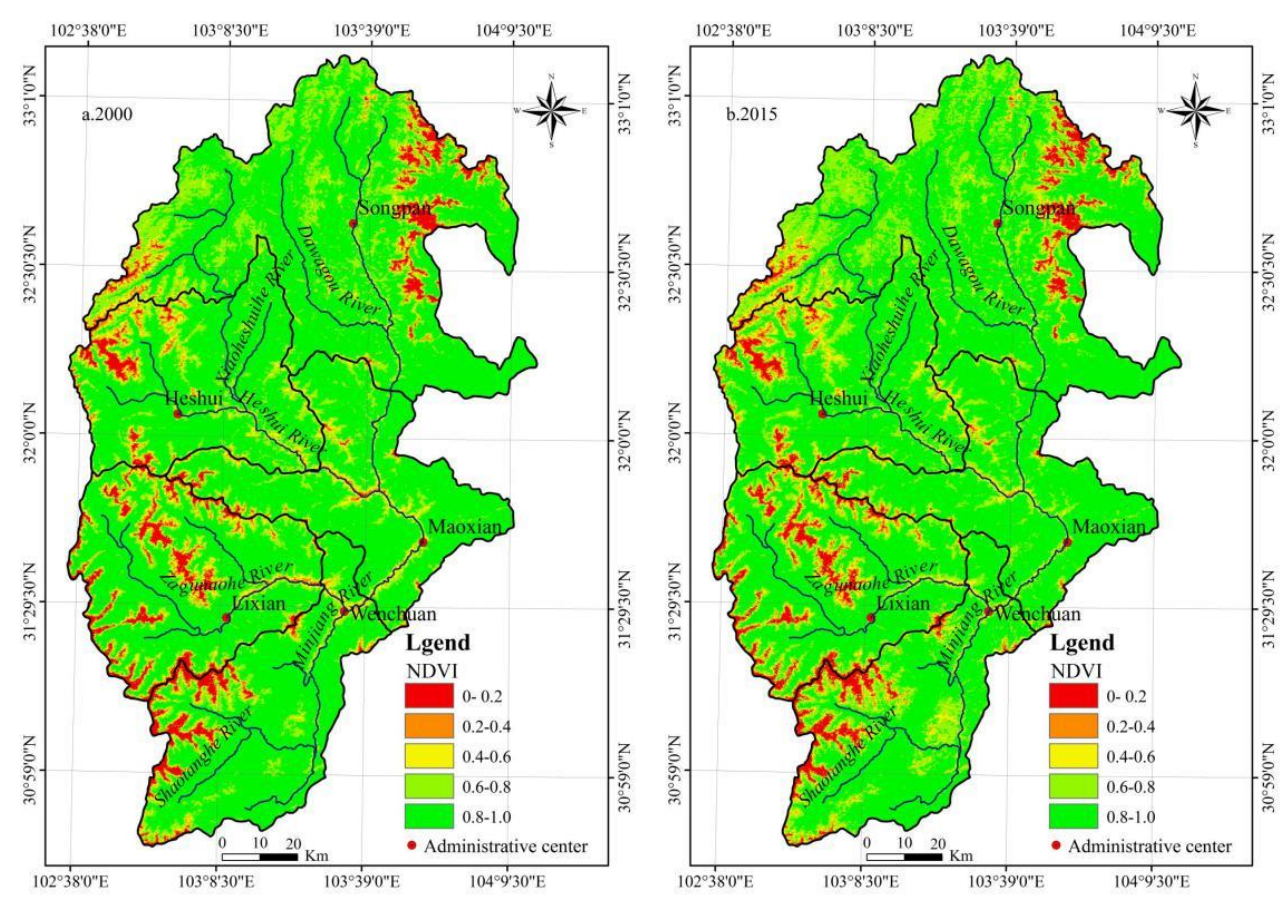

Fig.2 Spatial patterns of NDVI during 2000-2015

From 2000 to 2015, the spatial distribution of vegetation cover showed obvious variation.

The vegetation cover changes were dominated by the increase of vegetation cover $(0<\mathrm{NDVI}<0.8)$ in the Minjiang River and its tributaries, showing a strip-like distribution pattern. The vegetation cover in other regions increased and decreased in a canine-toothed pattern, and the vegetation cover areas with NDVI>0.8 had a decreasing trend (Fig.2, Fig.3). The region with NDVI greater than 0.8 accounted for more than $65 \%$, mainly distributed in shrubs, meadows, broad-leaved forests and mixed coniferous broad-leaved forests with elevations below $3600 \mathrm{~m}$ in the upper reaches of the Minjiang River. The areas of $0.6<\mathrm{NDVI}<0.8$ accounted for about $17 \%$, mainly distributed on the slopes on both sides of the Minjiang River and its tributaries, in the middle or low mountains of south-eastern Wenchuan County, in the middle or high mountains of north-western Wenchuan County and in the mountainous areas of north-western Songpan County. The areas of $0.2<\mathrm{NDVI}<0.6$ accounted for less than $9 \%$, mainly distributed on the banks of the Heishui River, the main stream of the upper Minjiang River and the Zagunao River, and at the edges of alpine vegetation zones in the western and north-eastern parts of the upper reaches of the Minjiang River. The areas with NDVI less than 0.2 only accounted for $4.5 \%$ or lower, mainly distributed in the extremely high-elevation regions in the western and north-eastern parts of the upper reaches of the Minjiang River with an elevation greater than $4500 \mathrm{~m}$. 
436

437

438

439

440

441

442

443

444

445

446

447

449
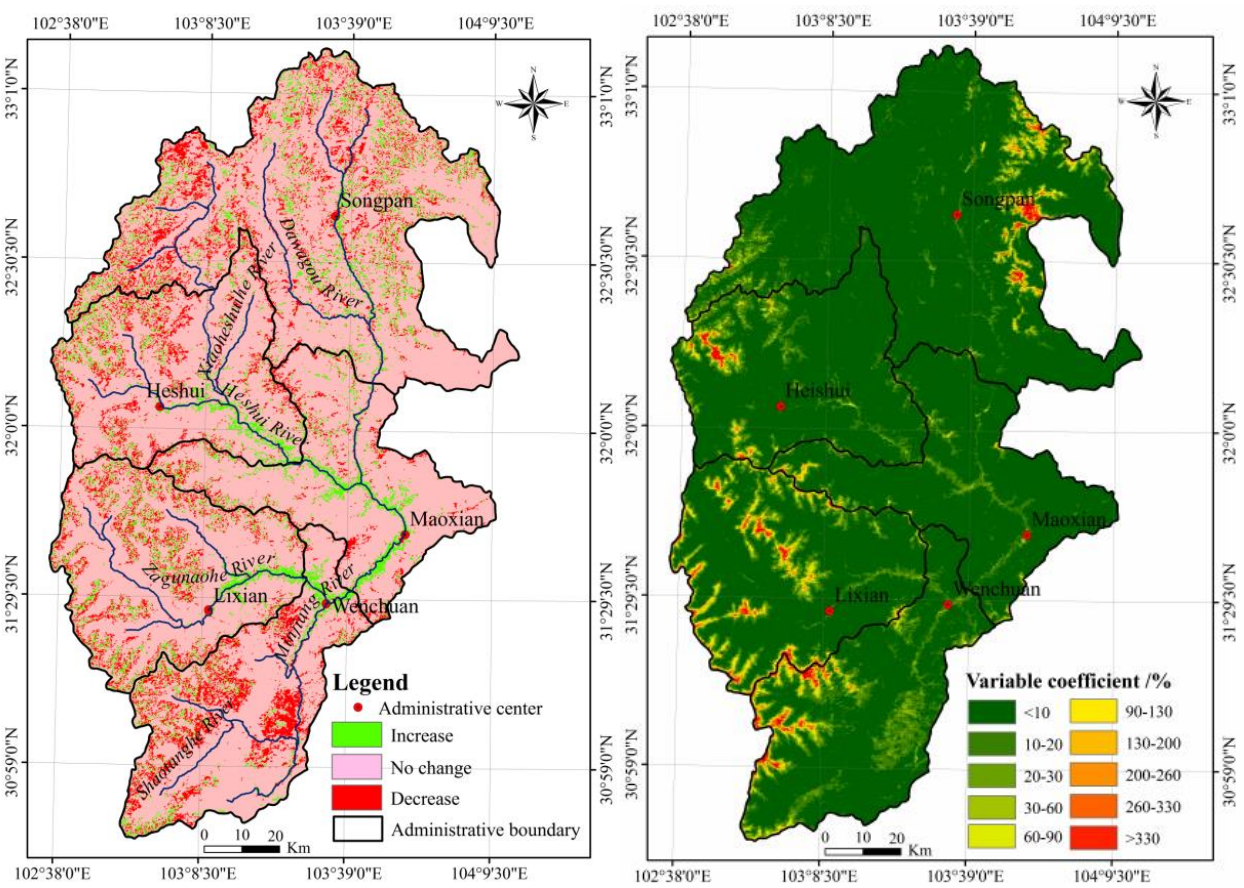

Fig.3 Spatial variation pattern of NDVI (right) and $C_{v}$ (left) during 2000 2015

The transfer matrix for different classes of NDVI was calculated based on the statistics of NDVI spatial distribution from 2000 to 2015 (Tab.3). From 2000 to 2015, the NDVI showed a significant transformation in the regions of NDVI $>0.6$, resulting in an increase in the areas of $0.6<\mathrm{NDVI}<0.8$ and a decrease in the areas of NDVI $>0.8$ (Tab.4). The roll-out areas of $0.6<\mathrm{NDVI}<0.8$ and NDVI $>0.8$ were $1680 \mathrm{~km}^{2}$ and $2080 \mathrm{~km}^{2}$, and the roll-in areas were $2415 \mathrm{~km}^{2}$ and $1185 \mathrm{~km}^{2}$, respectively, which led to the increase and decrease in the areas of $0.6<\mathrm{NDVI}<0.8$ and NDVI $>0.8$, respectively. Vegetation cover changes were dominated by the rise of vegetation cover $(0<\mathrm{NDVI}<0.8)$ in the Minjiang River and its tributaries, showing a strip-like pattern. The vegetation cover in other areas increased and decreased in a canine-toothed pattern.

\begin{tabular}{lccccccc}
\multicolumn{7}{c}{ Tab.3 Transfer matrix of NDVI changes during $2000 \sim 2015\left(\mathrm{~km}^{2}\right)$} \\
\hline NDVI & $(0 \sim 0.2)$ & {$[0.2 \sim 0.4)$} & {$[0.4 \sim 0.6]$} & {$[0.6 \sim 0.8)$} & {$[0.8 \sim 1)$} & 2015 Total & Transfer in \\
\hline$(0 \sim 0.2)$ & 17600.00 & 169.00 & 36.50 & 10.63 & 6.44 & 17800.00 & 222.56 \\
{$[0.2 \sim 0.4)$} & 156.00 & 395.00 & 247.00 & 52.63 & 12.81 & 864.00 & 468.44 \\
{$[0.4 \sim 0.6)$} & 37.25 & 215.00 & 670.00 & 497.00 & 93.06 & 1510.00 & 842.31 \\
{$[0.6 \sim 0.8)$} & 14.69 & 41.38 & 389.00 & 2670.00 & 1970.00 & 5080.00 & 2415.06 \\
{$[0.8 \sim 1.0)$} & 8.88 & 9.50 & 46.94 & 1120.00 & 15000.00 & 16200.00 & 1185.31 \\
2000 Total & 17800.00 & 830.00 & 1390.00 & 4350.00 & 17100.00 & & \\
Transfer out & 217.00 & 435.00 & 720.00 & 1680.00 & 2080.00 & & \\
change & 5.75 & 33.69 & 123.00 & 729.00 & -890.00 & & \\
\hline
\end{tabular}


The fluctuation of vegetation cover is relatively stable in the upper reaches of Minjiang River 452 from 2000 to 2015, with an average variable coefficient $\left(C_{v}\right)$ of $10.00 \%$ (Fig 3, right). The areas with $\mathrm{CV}<10 \%$ accounted for $79.02 \%$, which were mainly distributed in area with FVC $>0.8$ and their margins. The area between $10 \%<C v<60 \%$ accounted for $17.30 \%$, mainly distributed on both sides, in the southern, western and northeastern regions of Minjiang River. The area with $C v>$ $60 \%$ accounted for $3.68 \%$, which were mainly distributed in the very low vegetation coverage areas of high mountains and extremely high mountains.

\subsection{Influence analysis of detection factors}

The Geographical Detector model was used to discover the influence of impact factors on NDVI. By calculating the $q$ value of each impact factor, this study identified the influence exerted by each impact factor on NDVI (Tab.4). Tab.4 shows that the $q$ value of each impact factor was relatively stable from 2000 to 2015 , but there were different variation trends. The $q$ values of elevation, annual average temperature, soil type, geomorphic type, dryness index, vegetation type, accumulated temperature $\left(\geq 10^{\circ} \mathrm{C}\right)$ and distance from road showed a trend of increase, decrease and then increase. The $q$ values of annual precipitation and land use type first raised and then fell. The $q$ values of the remaining factors had relatively little variation.

According to Tab.4, the order of the influence of various impact factors on NDVI from 2000 to 2015 was: elevation > annual average temperature > soil type > geomorphic type > dryness index $>$ annual precipitation $>$ land use type $>$ accumulated temperature $\left(\geq 10^{\circ} \mathrm{C}\right)>$ vegetation type $>$ distance from road $>$ distance from township $>$ distance from river $>$ total radiation $>$ humidity index $>$ population density $>$ distance from county $>$ GDP density $>$ slope gradient $>$ slope aspect. 
Tab.4 The $q$ values of factors from 2010 to 2015

\begin{tabular}{ccccccccccc}
\hline Year & \multicolumn{2}{c}{2000} & \multicolumn{2}{c}{2005} & \multicolumn{2}{c}{2010} & \multicolumn{2}{c}{2015} & \multicolumn{2}{c}{ Mean value in 2000 2015 } \\
\hline Factors & $q$ & $p$ value & $q$ & $p$ value & $q$ & $p$ value & $q$ & $p$ value & $q$ & $p$ value \\
\hline$x_{1}$ & 0.0026 & 1.000 & 0.0099 & 0.000 & 0.0722 & 0.000 & 0.0132 & 0.000 & 0.0245 & 0.250 \\
$x_{2}$ & 0.0037 & 0.999 & 0.0100 & 0.000 & 0.0064 & 0.540 & 0.0208 & 0.000 & 0.0102 & 0.385 \\
$x_{3}$ & 0.1610 & 0.000 & 0.1675 & 0.000 & 0.1321 & 0.000 & 0.1579 & 0.000 & 0.1546 & 0.000 \\
$x_{4}$ & 0.0167 & 0.000 & 0.0191 & 0.000 & 0.0174 & 0.000 & 0.0199 & 0.000 & 0.0183 & 0.000 \\
$x_{5}$ & 0.0803 & 0.000 & 0.1050 & 0.000 & 0.0776 & 0.000 & 0.1044 & 0.000 & 0.0918 & 0.000 \\
$x_{6}$ & 0.1007 & 0.000 & 0.1306 & 0.000 & 0.1046 & 0.000 & 0.1264 & 0.000 & 0.1156 & 0.000 \\
$x_{7}$ & 0.0729 & 0.000 & 0.1050 & 0.000 & 0.0761 & 0.000 & 0.0902 & 0.000 & 0.0861 & 0.000 \\
$x_{8}$ & 0.5096 & 0.000 & 0.5714 & 0.000 & 0.4846 & 0.000 & 0.5389 & 0.000 & 0.5261 & 0.000 \\
$x_{9}$ & 0.1281 & 0.000 & 0.1591 & 0.000 & 0.1154 & 0.000 & 0.1505 & 0.000 & 0.1383 & 0.000 \\
$x_{10}$ & 0.1971 & 0.000 & 0.2346 & 0.000 & 0.1777 & 0.000 & 0.1707 & 0.000 & 0.1950 & 0.000 \\
$x_{11}$ & 0.0246 & 0.000 & 0.0334 & 0.000 & 0.0280 & 0.000 & 0.0332 & 0.000 & 0.0298 & 0.000 \\
$x_{12}$ & 0.1492 & 0.000 & 0.1913 & 0.000 & 0.1404 & 0.000 & 0.1786 & 0.000 & 0.1649 & 0.000 \\
$x_{13}$ & 0.0661 & 0.000 & 0.0713 & 0.000 & 0.0431 & 0.000 & 0.0716 & 0.000 & 0.0630 & 0.000 \\
$x_{14}$ & 0.1712 & 0.000 & 0.2210 & 0.000 & 0.1931 & 0.000 & 0.1954 & 0.000 & 0.1952 & 0.000 \\
$x_{15}$ & 0.5816 & 0.000 & 0.6420 & 0.000 & 0.5725 & 0.000 & 0.6064 & 0.000 & 0.6006 & 0.000 \\
$x_{16}$ & 0.0062 & 0.000 & 0.0057 & 0.000 & 0.0046 & 0.000 & 0.0087 & 0.000 & 0.0063 & 0.000 \\
$x_{17}$ & 0.0026 & 0.000 & 0.0031 & 0.000 & 0.0040 & 0.000 & 0.0046 & 0.000 & 0.0036 & 0.000 \\
$x_{18}$ & 0.1383 & 0.000 & 0.1556 & 0.000 & 0.1409 & 0.000 & 0.1438 & 0.000 & 0.1447 & 0.000 \\
$x_{19}$ & 0.3919 & 0.000 & 0.4194 & 0.000 & 0.3612 & 0.000 & 0.3881 & 0.000 & 0.3902 & 0.000 \\
\hline & & & & & & & & \\
\end{tabular}

\section{4}

According to Tab.4, the mean $q$ values of elevation, annual average temperature and soil type from 2000 to 2015 were the largest, reaching $0.6006,0.5261$ and 0.3902 or larger, respectively, and the explanatory power was $60 \%, 52 \%$ and $39 \%$ or larger, respectively. The mean $q$ values of geomorphic type and annual precipitation,were 0.1952 and 0.1950 , respectively, and their explanatory power was all above $19 \%$. The mean $q$ values ofdryness index, land use type, vegetation type, accumulated temperature $\left(\geq 10^{\circ} \mathrm{C}\right)$ and distance from road were $0.1649,0.1546$, $0.1447,0.1383$ and 0.1156 , respectively, and their explanatory power was all above $11 \%$. The $q$ values of the remaining factors were all less than 0.09. Although the influence of the explanatory power of a single factor, such as the distance from river, total radiation, humidity index, population density, distance from county, GDP density, slope gradient or slope aspect was small, its combination with other factors can have a relatively large impact on vegetation change. Therefore, the elevation, annual average temperature, soil type, geomorphic type and annual precipitation were the main factors influencing the vegetation change. 


\subsection{Indication analysis of detection factors}

Based on the Geographical Detector model, the suitable type or range of factors beneficial to vegetation growth is analyzed, and the statistical significance tests are passed at the $95 \%$ confidence level (Tab.5). The larger the NDVI value of vegetation, the more suitable the features of each natural factor are for vegetation growth. The difference of the mean vegetation NDVI value between different natural factors is significant (Tab.5).

\begin{tabular}{|c|c|c|}
\hline Factor & Suitable types or range & mean value of NDVI \\
\hline GDP density/yuan $/ \mathrm{km}^{2}$ & 77 104 & 0.8507 \\
\hline Density of population/person $/ \mathrm{km}^{2}$ & $28.11 \sim 33.18$ & 0.8693 \\
\hline Land use/types & forestland, grassland & 0.8655 \\
\hline Distance from county seat $/ \mathrm{m}$ & $0 \sim 7222.84$ & 0.8490 \\
\hline Distance from town $/ \mathrm{m}$ & $3600.33 \sim 6322.39$ & 0.8617 \\
\hline Distance from $\mathrm{road} / \mathrm{m}$ & $1558.93 \sim 3410.16$ & 0.8610 \\
\hline Distance from river/m & $1576.01 \sim 3327.13$ & 0.8621 \\
\hline Annual average temperature $/{ }^{\circ} \mathrm{C}$ & $6.3 \sim 7.95$ & 0.8843 \\
\hline Accumulated temperature $\left(\geq 10^{\circ} \mathrm{C}\right) /{ }^{\circ} \mathrm{C}$ & $15643 \sim 20428$ & 0.8878 \\
\hline Average annual precipitation $/ \mathrm{mm}$ & $796.65 \sim 817.49$ & 0.8570 \\
\hline Moisture index/level & $0.08 \sim 0.15$ & 0.8140 \\
\hline Index of aridity/level & 2 & 0.8571 \\
\hline Global radiation $/ \mathrm{MJ} / \mathrm{m}^{2}$ & $3888.40 \sim 4042.48$ & 0.8644 \\
\hline Geomorphic type/types & Hills、MIR & 0.8045 \\
\hline Elevation $/ \mathrm{m}$ & $2412 \sim 2748$ & 0.8850 \\
\hline Slope ${ }^{\circ}$ & $31.80 \sim 35.27$ & 0.8048 \\
\hline Aspect $/^{\circ}$ & $292.5 \sim 337.5$ & 0.8058 \\
\hline Vegetation type/types & $\mathrm{BLF}, \mathrm{COF}$ & 0.8667 \\
\hline Soil type/types & BCFS、YBS、YCS、BRS、GCS & 0.9390 \\
\hline
\end{tabular}

Note: MIR, minor relief mountain; BLF, broad-leaved forest; COF, coniferous forest; BCFS, brown coniferous forest soils; YBS,yellow brown soil; YCS, yellow clunamon soil; BRS, brown soil; GCS, gray cinnamonic soil.

With the increase of GDP density, the mean value of NDVI fluctuates around 0.8507 , and reaches the maximum of 0.8507 in the range of $77 \sim 104$ yuan $/ \mathrm{km}^{2}$. With the increase of population density, the mean NDVI shows a relatively large fluctuation, reaching a maximum value of 0.8693 at 28.11 33.188 people/ $\mathrm{km}^{2}$ and followed by a downward trend. With the different land use, the mean NDVI gets to its maximum of 0.8655 in grassland and woodland. The increase in the distance from county makes the mean NDVI to decrease first, fluctuate and then increase again. 
The maximum NDVI is 0.8490 at the distance of $0 \sim 7222.84 \mathrm{~m}$. With the increase of distance from township, road or river, the mean NDVI shows a downward trend and the maximum NDVI of 0.8617, 0.8610 and 0.8621 are reached at $3600.33 \sim 6322.39 \mathrm{~km}, 1558.93 \sim 3410.16 \mathrm{~km}$ and $1576.01 \sim 3327.13 \mathrm{~km}$, respectively. With the increase of annual average temperature, the mean NDVI shows a rising trend, getting to a maximum NDVI of 0.8843 at $6.3 \sim 7.95^{\circ} \mathrm{C}$. The increase of $\geq$ accumulated temperature $\left(\geq 10^{\circ} \mathrm{C}\right)$ and total radiation value give the mean NDVI a decreasing trend, and the maximum NDVI are 0.8878 and 0.8644 at $15643 \sim 20428^{\circ} \mathrm{C}$ and $3888.40 \sim 4042.48$ $\mathrm{MJ} / \mathrm{m}^{2}$, respectively. With the increase of annual precipitation, the mean NDVI fluctuates, increases, and then decreases, and the maximum NDVI of 0.8570 is reached at $796.65 \sim 817.49$ $\mathrm{mm}$. The mean NDVI fluctuates with the increase of humidity index and reaches the maximum NDVI of 0.8140 at level $8 \sim 15$. With the increase of dryness index, the mean NDVI shows a fluctuation trend of increase, decrease, increase and then decrease, and its maximum NDVI is 0.8571 at level 2. The mean NDVI has a slight fluctuation and then decreases with different geomorphic types and elevations. The maximum NDVI are 0.8045 and 0.8850 at hills or small relief mountains (low or middle mountains) and 2412 2748 m, respectively. The mean NDVI increases with the slope, showing a trend of increase first and then decrease. The maximum NDVI is 0.8048 on a slope of $31.80 \sim 35.27^{\circ}$. With the variation of slope aspect, the mean NDVI shows a fluctuation trend of decrease first and then increase, reaching a maximum NDVI of 0.8058 between the range 292.5 337.5 (northwest slope). The variation of mean NDVI shows different trends with different vegetation types and soil types. The maximum NDVI of 0.8667 and 0.9390 are reached in broad-leaved forest, coniferous forest and yellow brown soil, brown soil dark brown soil and cinnamon soil.

\subsection{Interaction analysis of detection factors}

Interaction detection mainly is used to identify the interaction of different candidate factors $\left(x_{i}\right)$ on the change of NDVI, analyze whether the explanatory power of the dependent variable NDVI is increased or decreased, or whether the effects of these factors on NDVI are independent. The study found that factors had interactive effects on NDVI in the upper reaches of Minjiang River, and the interaction between factors showed mutual enhancement and nonlinear enhancement, and there was no factor that acted independently (Tab.6). According to Table 6 , the $q$ values of interactions between most impact factors are higher 
than the $q$ values of any individual impact factors, and the interactive influence of impact factors is manifested as mutual enhancement effects and nonlinear enhancement effects.

The interactive influence of annual average temperature and soil type interact with elevation respectively are mutually enhanced and nonlinear enhancement effects, i.e, $x_{8} \cap x_{15}(0.628)>$ $x_{15} \cap x_{19}\left(0.623>x_{8} \cap x_{19}\right.$ (0.585) (Tab.6). The interactive influence of geomorphic types, dryness index, annual precipitation, land use type, vegetation type, accumulated temperature $\left(\geq 10^{\circ} \mathrm{C}\right)$ interact with the distance from road with elevation respectively show mutually enhanced and nonlinear enhancement effectsy (Tab.6), i.e, $x_{10} \cap x_{15}(0.637)>x_{14} \cap x_{15}(0.627)>x_{6} \cap x_{15}(0.620)$ $>x_{15} \cap x_{18}(0.612)$ or $x_{9} \cap x_{15}(0.612)>x_{12} \cap x_{15}(0.609)>x_{3} \cap x_{15}$ (0.237). The interactions between factors, i.e, GDP density, annual average precipitation, total radiation, population density, distance from river, slope direction, slope, vegetation type and elevation are mutually enhanced and nonlinear enhancement effects, such as $x_{1} \cap x_{15}\left(0.638>x_{10} \cap x_{15}(0.637)>x_{13} \cap x_{15}(0.629)>x_{2} \cap x_{15}\right.$ $(0.624)>x_{7} \cap x_{15}(0.619)>x_{15} \cap x_{17}(0.616)>x_{15} \cap x_{16}(0.614)>x_{15} \cap x_{18}(0.612)$. The interaction between soil type, annual average precipitation, land use, geomorphic type, humidity index, global radiation, distance from township, river, road, vegetation type, accumulated temperature $\left(\geq 10^{\circ} \mathrm{C}\right)$, and annual average temperature showed mutual enhancement and nonlinear enhancement (Tab.6), i.e, $x_{8} \cap x_{19}(0.585)>x_{8} \cap x_{10}(0.573) x_{3} \cap x_{8}(0.567)>x_{8} \cap x_{14}(0.566)>x_{8} \cap x_{11}(0.562)>x_{8} \cap$ $x_{13}(0.555)>x_{5} \cap x_{8} \quad(0.553)>x_{7} \cap x_{8}(0.552)$ or $x_{6} \cap x_{8}(0.552)>x_{8} \cap x_{18}(0.548)>x_{8} \cap x_{9} \quad(0.547)$ or $x_{8} \cap x_{16}(0.547)>x_{8} \cap x_{17}(0.546)>x_{8} \cap x_{12}(0.543), x_{14} \cap x_{19}\left(0.489>x_{4} \cap x_{19}\left(0.467>x_{6} \cap x_{19}\right.\right.$ $(0.429)>x_{3} \cap x_{19}(0.444)>x_{12} \cap x_{19}(0.428)>x_{11} \cap x_{19}(0.426)>x_{13} \cap x_{19}(0.412)>x_{18} \cap x_{19}$ (0.407) . Therefore, the impact factors interaction on NDVI is not a simple superposition process, but a mutual enhancement or nonlinear enhancement effect.

Tab.6 Interaction between factors that influence changes of NDVI

\begin{tabular}{|c|c|c|c|c|c|c|c|}
\hline $\mathrm{C}$ & $\mathrm{A}+\mathrm{B}$ & Result & Interpretation & $\mathrm{C}$ & $A+B$ & Result & Interpretation \\
\hline$x_{1} \cap x_{2}=0.032$ & $<0.034=x_{1}+x_{2}$ & $\mathrm{C}<\mathrm{A}+\mathrm{B}$ & $\uparrow$ & $x_{6} \cap x_{13}=0.210$ & $>0.198=x_{6}+x_{13}$ & $\mathrm{C}>\mathrm{A}+\mathrm{B}$ & $\uparrow \uparrow$ \\
\hline$x_{1} \cap x_{3}=0.188$ & $>0.171=x_{1}+x_{3}$ & $\mathrm{C}>\mathrm{A}+\mathrm{B}$ & $\uparrow \uparrow$ & $x_{6} \cap x_{14}=0.261$ & $<0.322=x_{6}+x_{14}$ & $\mathrm{C}<\mathrm{A}+\mathrm{B}$ & $\uparrow$ \\
\hline$x_{1} \cap x_{4}=0.071$ & $>0.033=x_{1}+x_{4}$ & $\mathrm{C}>\mathrm{A}+\mathrm{B}$ & $\uparrow \uparrow$ & $x_{6} \cap x_{15}=0.620$ & $<0.733=x_{6}+x_{15}$ & $\mathrm{C}<\mathrm{A}+\mathrm{B}$ & $\uparrow$ \\
\hline$x_{1} \cap x_{5}=0.148$ & $>0.118=x_{1}+x_{5}$ & $\mathrm{C}>\mathrm{A}+\mathrm{B}$ & $\uparrow \uparrow$ & $x_{6} \cap x_{16}=0.142$ & $>0.135=x_{6}+x_{16}$ & $\mathrm{C}>\mathrm{A}+\mathrm{B}$ & $\uparrow \uparrow$ \\
\hline$x_{1} \cap x_{6}=0.173$ & $>0.140=x_{1}+x_{6}$ & $\mathrm{C}>\mathrm{A}+\mathrm{B}$ & $\uparrow \uparrow$ & $x_{6} \cap x_{17}=0.137$ & $>0.130=x_{6}+x_{17}$ & $\mathrm{C}>\mathrm{A}+\mathrm{B}$ & $\uparrow \uparrow$ \\
\hline$x_{1} \cap x_{7}=0.137$ & $>0.103=x_{1}+x_{7}$ & $\mathrm{C}>\mathrm{A}+\mathrm{B}$ & $\uparrow \uparrow$ & $x_{6} \cap x_{18}=0.226$ & $<0.270=x_{6}+x_{18}$ & $\mathrm{C}<\mathrm{A}+\mathrm{B}$ & $\uparrow$ \\
\hline$x_{1} \cap x_{8}=0.567$ & $>0.552=x_{1}+x_{8}$ & $\mathrm{C}>\mathrm{A}+\mathrm{B}$ & $\uparrow \uparrow$ & $x_{6} \cap x_{19}=0.429$ & $<0.515=x_{6}+x_{19}$ & $\mathrm{C}<\mathrm{A}+\mathrm{B}$ & $\uparrow$ \\
\hline$x_{1} \cap x_{9}=0.182$ & $>0.164=x_{1}+x_{9}$ & $\mathrm{C}>\mathrm{A}+\mathrm{B}$ & $\uparrow \uparrow$ & $x_{7} \cap x_{8}=0.552$ & $<0.629=x_{7}+x_{8}$ & $\mathrm{C}<\mathrm{A}+\mathrm{B}$ & $\uparrow$ \\
\hline
\end{tabular}




\begin{tabular}{|c|c|c|c|c|c|c|}
\hline$x_{1} \cap x_{10}=0.249$ & $<0.184=x_{1}+x_{10}$ & $\mathrm{C}>\mathrm{A}+\mathrm{B}$ & $\uparrow \uparrow$ & $x_{7} \cap x_{9}=0.201$ & $<0.241=x_{7}+x_{9}$ & $\mathrm{C}<\mathrm{A}+\mathrm{B}$ \\
\hline$x_{1} \cap x_{11}=0.099$ & $>0.046=x_{1}+x_{11}$ & $\mathrm{C}>\mathrm{A}+\mathrm{B}$ & $\uparrow \uparrow$ & $x_{7} \cap x_{10}=0.247$ & $<0.261=x_{7}+x_{10}$ & $\mathrm{C}<\mathrm{A}+\mathrm{B}$ \\
\hline$x_{1} \cap x_{12}=0.203$ & $>0.192=x_{1}+x_{12}$ & $\mathrm{C}>\mathrm{A}+\mathrm{B}$ & $\uparrow \uparrow$ & $x_{7} \cap x_{11}=0.171$ & $>0.123=x_{7}+x_{11}$ & $\mathrm{C}>\mathrm{A}+\mathrm{B}$ \\
\hline$x_{1} \cap x_{13}=0.178$ & $>0.085=x_{1}+x_{13}$ & $\mathrm{C}>\mathrm{A}+\mathrm{B}$ & $\uparrow \uparrow$ & $x_{7} \cap x_{12}=0.216$ & $<0.269=x_{7}+x_{12}$ & $\mathrm{C}<\mathrm{A}+\mathrm{B}$ \\
\hline$x_{1} \cap x_{14}=0.268$ & $>0.209=x_{1}+x_{14}$ & $\mathrm{C}>\mathrm{A}+\mathrm{B}$ & $\uparrow \uparrow$ & $x_{7} \cap x_{13}=0.182$ & $>0.162=x_{7}+x_{13}$ & $\mathrm{C}>\mathrm{A}+\mathrm{B}$ \\
\hline$x_{1} \cap x_{15}=0.638$ & $>0.620=x_{1}+x_{15}$ & $\mathrm{C}>\mathrm{A}+\mathrm{B}$ & $\uparrow \uparrow$ & $x_{7} \cap x_{14}=0.241$ & $<0.286=x_{7}+x_{14}$ & $\mathrm{C}<\mathrm{A}+\mathrm{B}$ \\
\hline$x_{1} \cap x_{16}=0.031$ & $>0.022=x_{1}+x_{16}$ & $\mathrm{C}>\mathrm{A}+\mathrm{B}$ & $\uparrow \uparrow$ & $x_{7} \cap x_{15}=0.619$ & $<0.697=x_{7}+x_{15}$ & $\mathrm{C}<\mathrm{A}+\mathrm{B}$ \\
\hline$x_{1} \cap x_{17}=0.023$ & $>0.018=x_{1}+x_{17}$ & $\mathrm{C}>\mathrm{A}+\mathrm{B}$ & $\uparrow \uparrow$ & $x_{7} \cap x_{16}=0.103$ & $>0.099=x_{7}+x_{16}$ & $\mathrm{C}>\mathrm{A}+\mathrm{B}$ \\
\hline$x_{1} \cap x_{18}=0.173$ & $>0.157=x_{1}+x_{18}$ & $\mathrm{C}>\mathrm{A}+\mathrm{B}$ & $\uparrow \uparrow$ & $x_{7} \cap x_{17}=0.101$ & $>0.095=x_{7}+x_{17}$ & $\mathrm{C}>\mathrm{A}+\mathrm{B}$ \\
\hline$x_{1} \cap x_{19}=0.419$ & $>0.401=x_{1}+x_{19}$ & $\mathrm{C}>\mathrm{A}+\mathrm{B}$ & $\uparrow \uparrow$ & $x_{7} \cap x_{18}=0.199$ & $<0.234=x_{7}+x_{18}$ & $\mathrm{C}<\mathrm{A}+\mathrm{B}$ \\
\hline$x_{2} \cap x_{3}=0.208$ & $>0.179=x_{2}+x_{3}$ & $\mathrm{C}>\mathrm{A}+\mathrm{B}$ & $\uparrow \uparrow$ & $x_{7} \cap x_{19}=0.411$ & $<0.478=x_{7}+x_{19}$ & $\mathrm{C}<\mathrm{A}+\mathrm{B}$ \\
\hline$x_{2} \cap x_{4}=0.086$ & $>0.041=x_{2}+x_{4}$ & $\mathrm{C}>\mathrm{A}+\mathrm{B}$ & $\uparrow \uparrow$ & $x_{8} \cap x_{9}=0.547$ & $<0.689=x_{8}+x_{9}$ & $\mathrm{C}<\mathrm{A}+\mathrm{B}$ \\
\hline$x_{2} \cap x_{5}=0.155$ & $>0.125=x_{2}+x_{5}$ & $\mathrm{C}>\mathrm{A}+\mathrm{B}$ & $\uparrow \uparrow$ & $x_{8} \cap x_{10}=0.573$ & $<0.710=x_{8}+x_{10}$ & $\mathrm{C}<\mathrm{A}+\mathrm{B}$ \\
\hline$x_{2} \cap x_{6}=0.185$ & $>0.147=x_{2}+x_{6}$ & $\mathrm{C}<\mathrm{A}+\mathrm{B}$ & $\uparrow \uparrow$ & $x_{8} \cap x_{11}=0.562$ & $<0.572=x_{8}+x_{11}$ & $\mathrm{C}<\mathrm{A}+\mathrm{B}$ \\
\hline$x_{2} \cap x_{7}=0.152$ & $>0.111=x_{2}+x_{7}$ & $\mathrm{C}>\mathrm{A}+\mathrm{B}$ & $\uparrow \uparrow$ & $x_{8} \cap x_{12}=0.543$ & $<0.718=x_{8}+x_{12}$ & $\mathrm{C}<\mathrm{A}+\mathrm{B}$ \\
\hline$x_{2} \cap x_{8}=0.557$ & $<0.560=x_{2}+x_{8}$ & $\mathrm{C}<\mathrm{A}+\mathrm{B}$ & $\uparrow$ & $x_{8} \cap x_{13}=0.555$ & $<0.611=x_{8}+x_{13}$ & $\mathrm{C}<\mathrm{A}+\mathrm{B}$ \\
\hline$x_{2} \cap x_{9}=0.191$ & $>0.171=x_{2}+x_{9}$ & $\mathrm{C}>\mathrm{A}+\mathrm{B}$ & $\uparrow \uparrow$ & $x_{8} \cap x_{14}=0.566$ & $<0.734=x_{8}+x_{14}$ & $\mathrm{C}<\mathrm{A}+\mathrm{B}$ \\
\hline$x_{2} \cap x_{10}=0.260$ & $>0.191=x_{2}+x_{10}$ & $\mathrm{C}>\mathrm{A}+\mathrm{B}$ & $\uparrow \uparrow$ & $x_{8} \cap x_{15}=0.628$ & $<0.95=x_{8}+x_{15}$ & $\mathrm{C}<\mathrm{A}+\mathrm{B}$ \\
\hline$x_{2} \cap x_{11}=0.108$ & $>0.054=x_{2}+x_{11}$ & $\mathrm{C}>\mathrm{A}+\mathrm{B}$ & $\uparrow \uparrow$ & $x_{8} \cap x_{16}=0.547$ & $<0.548=x_{8}+x_{16}$ & $\mathrm{C}<\mathrm{A}+\mathrm{B}$ \\
\hline$x_{2} \cap x_{12}=0.215$ & $>0.199=x_{2}+x_{12}$ & $\mathrm{C}>\mathrm{A}+\mathrm{B}$ & $\uparrow \uparrow$ & $x_{8} \cap x_{17}=0.546$ & $>0.543=x_{8}+x_{17}$ & $\mathrm{C}>\mathrm{A}+\mathrm{B}$ \\
\hline$x_{2} \cap x_{13}=0.226$ & $>0.092=x_{2}+x_{13}$ & $\mathrm{C}>\mathrm{A}+\mathrm{B}$ & $\uparrow \uparrow$ & $x_{8} \cap x_{18}=0.548$ & $<0.683=x_{8}+x_{18}$ & $\mathrm{C}<\mathrm{A}+\mathrm{B}$ \\
\hline$x_{2} \cap x_{14}=0.257$ & $>0.216=x_{2}+x_{14}$ & $\mathrm{C}>\mathrm{A}+\mathrm{B}$ & $\uparrow \uparrow$ & $x_{8} \cap x_{19}=0.585$ & $<0.927=x_{8}+x_{19}$ & $\mathrm{C}<\mathrm{A}+\mathrm{B}$ \\
\hline$x_{2} \cap x_{15}=0.624$ & $<0.627=x_{2}+x_{15}$ & $\mathrm{C}<\mathrm{A}+\mathrm{B}$ & $\uparrow$ & $x_{9} \cap x_{10}=0.267$ & $<0.321=x_{9}+x_{10}$ & $\mathrm{C}<\mathrm{A}+\mathrm{B}$ \\
\hline$x_{2} \cap x_{16}=0.265$ & $>0.238=x_{2}+x_{16}$ & $\mathrm{C}>\mathrm{A}+\mathrm{B}$ & $\uparrow \uparrow$ & $x_{9} \cap x_{11}=0.193$ & $>0.184=x_{9}+x_{11}$ & $\mathrm{C}>\mathrm{A}+\mathrm{B}$ \\
\hline$x_{2} \cap x_{17}=0.290$ & $>0.025=x_{2}+x_{17}$ & $\mathrm{C}>\mathrm{A}+\mathrm{B}$ & $\uparrow \uparrow$ & $x_{9} \cap x_{12}=0.185$ & $<0.329=x_{9}+x_{12}$ & $\mathrm{C}<\mathrm{A}+\mathrm{B}$ \\
\hline$x_{2} \cap x_{18}=0.181$ & $>0.165=x_{2}+x_{18}$ & $\mathrm{C}>\mathrm{A}+\mathrm{B}$ & $\uparrow \uparrow$ & $x_{9} \cap x_{13}=0.194$ & $<0.222=x_{9}+x_{13}$ & $\mathrm{C}<\mathrm{A}+\mathrm{B}$ \\
\hline$x_{2} \cap x_{19}=0.427$ & $>0.409=x_{2}+x_{19}$ & $\mathrm{C}>\mathrm{A}+\mathrm{B}$ & $\uparrow \uparrow$ & $x_{9} \cap x_{14}=0.297$ & $<0.346=x_{9}+x_{14}$ & $\mathrm{C}<\mathrm{A}+\mathrm{B}$ \\
\hline$x_{3} \cap x_{4}=0.182$ & $>0.178=x_{3}+x_{4}$ & $\mathrm{C}>\mathrm{A}+\mathrm{B}$ & $\uparrow \uparrow$ & $x_{9} \cap x_{15}=0.612$ & $<0.757=x_{9}+x_{15}$ & $\mathrm{C}<\mathrm{A}+\mathrm{B}$ \\
\hline$x_{3} \cap x_{5}=0.237$ & $<0.262=x_{3}+x_{5}$ & $\mathrm{C}<\mathrm{A}+\mathrm{B}$ & $\uparrow$ & $x_{9} \cap x_{16}=0.158$ & $<0.159=x_{9}+x_{16}$ & $\mathrm{C}<\mathrm{A}+\mathrm{B}$ \\
\hline$x_{3} \cap x_{6}=0.254$ & $<0.284=x_{3}+x_{6}$ & $\mathrm{C}<\mathrm{A}+\mathrm{B}$ & $\uparrow$ & $x_{9} \cap x_{17}=0.159$ & $>0.155=x_{9}+x_{17}$ & $\mathrm{C}>\mathrm{A}+\mathrm{B}$ \\
\hline$x_{3} \cap x_{7}=0.234$ & $>0.248=x_{3}+x_{7}$ & $\mathrm{C}<\mathrm{A}+\mathrm{B}$ & $\uparrow$ & $x_{9} \cap x_{18}=0.239$ & $<0.294=x_{9}+x_{18}$ & $\mathrm{C}<\mathrm{A}+\mathrm{B}$ \\
\hline$x_{3} \cap x_{8}=0.567$ & $<0.697=x_{3}+x_{8}$ & $\mathrm{C}<\mathrm{A}+\mathrm{B}$ & $\uparrow$ & $x_{9} \cap x_{19}=0.430$ & $<0.539=x_{9}+x_{19}$ & $\mathrm{C}<\mathrm{A}+\mathrm{B}$ \\
\hline$x_{3} \cap x_{9}=0.271$ & $<0.308=x_{3}+x_{9}$ & $\mathrm{C}<\mathrm{A}+\mathrm{B}$ & $\uparrow$ & $x_{10} \cap x_{11}=0.369$ & $>0.204=x_{10}+x_{11}$ & $\mathrm{C}>\mathrm{A}+\mathrm{B}$ \\
\hline$x_{3} \cap x_{10}=0.295$ & $<0.329=x_{3}+x_{10}$ & $\mathrm{C}<\mathrm{A}+\mathrm{B}$ & $\uparrow$ & $x_{10} \cap x_{12}=0.282$ & $<0.349=x_{10}+x_{12}$ & $\mathrm{C}<\mathrm{A}+\mathrm{B}$ \\
\hline$x_{3} \cap x_{11}=0.198$ & $>0.191=x_{3}+x_{11}$ & $\mathrm{C}>\mathrm{A}+\mathrm{B}$ & $\uparrow \uparrow$ & $x_{10} \cap x_{13}=0.326$ & $>0.242=x_{10}+x_{13}$ & $\mathrm{C}>\mathrm{A}+\mathrm{B}$ \\
\hline$x_{3} \cap x_{12}=0.293$ & $<0.337=x_{3}+x_{12}$ & $\mathrm{C}<\mathrm{A}+\mathrm{B}$ & $\uparrow$ & $x_{10} \cap x_{14}=0.275$ & $<0.366=x_{10}+x_{14}$ & $\mathrm{C}<\mathrm{A}+\mathrm{B}$ \\
\hline$x_{3} \cap x_{13}=0.216$ & $<0.230=x_{3}+x_{13}$ & $\mathrm{C}<\mathrm{A}+\mathrm{B}$ & $\uparrow$ & $x_{10} \cap x_{15}=0.637$ & $<0.777=x_{10}+x_{15}$ & $\mathrm{C}<\mathrm{A}+\mathrm{B}$ \\
\hline$x_{3} \cap x_{14}=0.333$ & $<0.353=x_{3}+x_{14}$ & $\mathrm{C}<\mathrm{A}+\mathrm{B}$ & $\uparrow$ & $x_{10} \cap x_{16}=0.191$ & $>0.179=x_{10}+x_{16}$ & $\mathrm{C}>\mathrm{A}+\mathrm{B}$ \\
\hline$x_{3} \cap x_{15}=0.628$ & $<0.764=x_{3}+x_{15}$ & $\mathrm{C}<\mathrm{A}+\mathrm{B}$ & $\uparrow$ & $x_{10} \cap x_{17}=0.180$ & $>0.175=x_{10}+x_{17}$ & $\mathrm{C}>\mathrm{A}+\mathrm{B}$ \\
\hline$x_{3} \cap x_{16}=0.164$ & $<0.167=x_{3}+x_{16}$ & $\mathrm{C}<\mathrm{A}+\mathrm{B}$ & $\uparrow$ & $x_{10} \cap x_{18}=0.278$ & $<0.314=x_{10}+x_{18}$ & $\mathrm{C}<\mathrm{A}+\mathrm{B}$ \\
\hline$x_{3} \cap x_{17}=0.166$ & $>0.162=x_{3}+x_{17}$ & $\mathrm{C}>\mathrm{A}+\mathrm{B}$ & $\uparrow \uparrow$ & $x_{10} \cap x_{19}=0.467$ & $<0.559=x_{10}+x_{19}$ & $\mathrm{C}<\mathrm{A}+\mathrm{B}$ \\
\hline$x_{3} \cap x_{18}=0.254$ & $<0.302=x_{3}+x_{18}$ & $\mathrm{C}<\mathrm{A}+\mathrm{B}$ & $\uparrow$ & $x_{11} \cap x_{12}=0.211$ & $<0.212=x_{11}+x_{12}$ & $\mathrm{C}<\mathrm{A}+\mathrm{B}$ \\
\hline
\end{tabular}




\begin{tabular}{|c|c|c|c|c|c|c|}
\hline$x_{3} \cap x_{19}=0.444$ & $<0.546=x_{3}+x_{19}$ & $\mathrm{C}<\mathrm{A}+\mathrm{B}$ & $\uparrow$ & $x_{11} \cap x_{13}=0.175$ & $>0.105=x_{11}+x_{13}$ & $\mathrm{C}>\mathrm{A}+\mathrm{B}$ \\
\hline$x_{4} \cap x_{5}=0.142$ & $>0.124=x_{4}+x_{5}$ & $\mathrm{C}>\mathrm{A}+\mathrm{B}$ & $\uparrow \uparrow$ & $x_{11} \cap x_{14}=0.259$ & $>0.229=x_{11}+x_{14}$ & $\mathrm{C}>\mathrm{A}+\mathrm{B}$ \\
\hline$x_{4} \cap x_{6}=0.173$ & $>0.146=x_{4}+x_{6}$ & $\mathrm{C}>\mathrm{A}+\mathrm{B}$ & $\uparrow \uparrow$ & $x_{11} \cap x_{15}=0.631$ & $<0.640=x_{11}+x_{15}$ & $\mathrm{C}<\mathrm{A}+\mathrm{B}$ \\
\hline$x_{4} \cap x_{7}=0.133$ & $>0.110=x_{4}+x_{7}$ & $\mathrm{C}>\mathrm{A}+\mathrm{B}$ & $\uparrow \uparrow$ & $x_{11} \cap x_{16}=0.049$ & $>0.042=x_{11}+x_{16}$ & $\mathrm{C}>\mathrm{A}+\mathrm{B}$ \\
\hline$x_{4} \cap x_{8}=0.552$ & $<0.556=x_{4}+x_{8}$ & $\mathrm{C}<\mathrm{A}+\mathrm{B}$ & $\uparrow$ & $x_{11} \cap x_{17}=0.042$ & $>0.038=x_{11}+x_{17}$ & $\mathrm{C}>\mathrm{A}+\mathrm{B}$ \\
\hline$x_{4} \cap x_{9}=0.178$ & $>0.170=x_{4}+x_{9}$ & $\mathrm{C}>\mathrm{A}+\mathrm{B}$ & $\uparrow \uparrow$ & $x_{11} \cap x_{18}=0.178$ & $>0.177=x_{11}+x_{18}$ & $\mathrm{C}>\mathrm{A}+\mathrm{B}$ \\
\hline$x_{4} \cap x_{10}=0.264$ & $>0.191=x_{4}+x_{10}$ & $\mathrm{C}>\mathrm{A}+\mathrm{B}$ & $\uparrow \uparrow$ & $x_{11} \cap x_{19}=0.426$ & $>0.421=x_{11}+x_{19}$ & $\mathrm{C}>\mathrm{A}+\mathrm{B}$ \\
\hline$x_{4} \cap x_{11}=0.086$ & $>0.053=x_{4}+x_{11}$ & $\mathrm{C}>\mathrm{A}+\mathrm{B}$ & $\uparrow \uparrow$ & $x_{12} \cap x_{13}=0.218$ & $<0.250=x_{12}+x_{13}$ & $\mathrm{C}<\mathrm{A}+\mathrm{B}$ \\
\hline$x_{4} \cap x_{12}=0.202$ & $>0.119=x_{4}+x_{12}$ & $\mathrm{C}>\mathrm{A}+\mathrm{B}$ & $\uparrow \uparrow$ & $x_{12} \cap x_{14}=0.304$ & $<0.374=x_{12}+x_{14}$ & $\mathrm{C}<\mathrm{A}+\mathrm{B}$ \\
\hline$x_{4} \cap x_{13}=0.118$ & $>0.092=x_{4}+x_{13}$ & $C>A+B$ & $\uparrow \uparrow$ & $x_{12} \cap x_{15}=0.609$ & $<0.785=x_{12}+x_{15}$ & $\mathrm{C}<\mathrm{A}+\mathrm{B}$ \\
\hline$x_{4} \cap x_{14}=0.230$ & $>0.053=x_{4}+x_{14}$ & $\mathrm{C}>\mathrm{A}+\mathrm{B}$ & $\uparrow \uparrow$ & $x_{12} \cap x_{16}=0.185$ & $<0.187=x_{12}+x_{16}$ & $\mathrm{C}<\mathrm{A}+\mathrm{B}$ \\
\hline$x_{4} \cap x_{15}=0.619$ & $<0.626=x_{4}+x_{15}$ & $\mathrm{C}<\mathrm{A}+\mathrm{B}$ & $\uparrow$ & $x_{12} \cap x_{17}=0.185$ & $>0.183=x_{12}+x_{17}$ & $\mathrm{C}>\mathrm{A}+\mathrm{B}$ \\
\hline$x_{4} \cap x_{16}=0.037$ & $>0.029=x_{4}+x_{16}$ & $C>A+B$ & $\uparrow \uparrow$ & $x_{12} \cap x_{18}=0.260$ & $<0.322=x_{12}+x_{18}$ & $\mathrm{C}<\mathrm{A}+\mathrm{B}$ \\
\hline$x_{4} \cap x_{17}=0.028$ & $>0.024=x_{4}+x_{17}$ & $\mathrm{C}>\mathrm{A}+\mathrm{B}$ & $\uparrow \uparrow$ & $x_{12} \cap x_{19}=0.428$ & $<0.567=x_{12}+x_{19}$ & $\mathrm{C}<\mathrm{A}+\mathrm{B}$ \\
\hline$x_{4} \cap x_{18}=0.171$ & $>0.164=x_{4}+x_{18}$ & $\mathrm{C}>\mathrm{A}+\mathrm{B}$ & $\uparrow \uparrow$ & $x_{13} \cap x_{14}=0.313$ & $>0.267=x_{13}+x_{14}$ & $\mathrm{C}>\mathrm{A}+\mathrm{B}$ \\
\hline$x_{4} \cap x_{19}=0.406$ & $<0.408=x_{4}+x_{19}$ & $\mathrm{C}<\mathrm{A}+\mathrm{B}$ & $\uparrow$ & $x_{13} \cap x_{15}=0.629$ & $<0.678=x_{13}+x_{15}$ & $\mathrm{C}<\mathrm{A}+\mathrm{B}$ \\
\hline$x_{5} \cap x_{6}=0.176$ & $<0.231=x_{5}+x_{6}$ & $\mathrm{C}<\mathrm{A}+\mathrm{B}$ & $\uparrow$ & $x_{13} \cap x_{16}=0.084$ & $>0.080=x_{13}+x_{16}$ & $\mathrm{C}>\mathrm{A}+\mathrm{B}$ \\
\hline$x_{5} \cap x_{7}=0.173$ & $<0.194=x_{5}+x_{7}$ & $\mathrm{C}<\mathrm{A}+\mathrm{B}$ & $\uparrow$ & $x_{13} \cap x_{17}=0.081$ & $>0.076=x_{13}+x_{17}$ & $\mathrm{C}>\mathrm{A}+\mathrm{B}$ \\
\hline$x_{5} \cap x_{8}=0.553$ & $<0.643=x_{5}+x_{8}$ & $\mathrm{C}<\mathrm{A}+\mathrm{B}$ & $\uparrow$ & $x_{13} \cap x_{18}=0.203$ & $<0.215=x_{13}+x_{18}$ & $\mathrm{C}<\mathrm{A}+\mathrm{B}$ \\
\hline$x_{5} \cap x_{9}=0.209$ & $<0.255=x_{5}+x_{9}$ & $\mathrm{C}<\mathrm{A}+\mathrm{B}$ & $\uparrow$ & $x_{13} \cap x_{19}=0.412$ & $<0.460=x_{13}+x_{19}$ & $\mathrm{C}<\mathrm{A}+\mathrm{B}$ \\
\hline$x_{5} \cap x_{10}=0.277$ & $>0.275=x_{5}+x_{10}$ & $\mathrm{C}>\mathrm{A}+\mathrm{B}$ & $\uparrow \uparrow$ & $x_{14} \cap x_{15}=0.627$ & $<0.802=x_{14}+x_{15}$ & $\mathrm{C}<\mathrm{A}+\mathrm{B}$ \\
\hline$x_{5} \cap x_{11}=0.150$ & $>0.138=x_{5}+x_{11}$ & $\mathrm{C}>\mathrm{A}+\mathrm{B}$ & $\uparrow \uparrow$ & $x_{14} \cap x_{16}=0.211$ & $>0.204=x_{14}+x_{16}$ & $\mathrm{C}>\mathrm{A}+\mathrm{B}$ \\
\hline$x_{5} \cap x_{12}=0.237$ & $<0.283=x_{5}+x_{12}$ & $\mathrm{C}<\mathrm{A}+\mathrm{B}$ & $\uparrow$ & $x_{14} \cap x_{17}=0.204$ & $>0.200=x_{14}+x_{17}$ & $\mathrm{C}>\mathrm{A}+\mathrm{B}$ \\
\hline$x_{5} \cap x_{13}=0.192$ & $>0.176=x_{5}+x_{13}$ & $\mathrm{C}>\mathrm{A}+\mathrm{B}$ & $\uparrow \uparrow$ & $x_{14} \cap x_{18}=0.299$ & $<0.339=x_{14}+x_{18}$ & $\mathrm{C}<\mathrm{A}+\mathrm{B}$ \\
\hline$x_{5} \cap x_{14}=0.262$ & $<0.300=x_{5}+x_{14}$ & $\mathrm{C}<\mathrm{A}+\mathrm{B}$ & $\uparrow$ & $x_{14} \cap x_{19}=0.489$ & $<0.583=x_{14}+x_{19}$ & $\mathrm{C}<\mathrm{A}+\mathrm{B}$ \\
\hline$x_{5} \cap x_{15}=0.619$ & $<0.711=x_{5}+x_{15}$ & $\mathrm{C}<\mathrm{A}+\mathrm{B}$ & $\uparrow$ & $x_{15} \cap x_{16}=0.614$ & $<0.615=x_{15}+x_{16}$ & $\mathrm{C}<\mathrm{A}+\mathrm{B}$ \\
\hline$x_{5} \cap x_{16}=0.117$ & $>0.113=x_{5}+x_{16}$ & $\mathrm{C}>\mathrm{A}+\mathrm{B}$ & $\uparrow \uparrow$ & $x_{15} \cap x_{17}=0.616$ & $>0.611=x_{15}+x_{17}$ & $\mathrm{C}>\mathrm{A}+\mathrm{B}$ \\
\hline$x_{5} \cap x_{17}=0.117$ & $>0.109=x_{5}+x_{17}$ & $\mathrm{C}>\mathrm{A}+\mathrm{B}$ & $\uparrow \uparrow$ & $x_{15} \cap x_{18}=0.612$ & $<0.750=x_{15}+x_{18}$ & $\mathrm{C}<\mathrm{A}+\mathrm{B}$ \\
\hline$x_{5} \cap x_{18}=0.214$ & $<0.248=x_{5}+x_{18}$ & $\mathrm{C}<\mathrm{A}+\mathrm{B}$ & $\uparrow$ & $x_{15} \cap x_{19}=0.623$ & $<0.994=x_{15}+x_{19}$ & $\mathrm{C}<\mathrm{A}+\mathrm{B}$ \\
\hline$x_{5} \cap x_{19}=0.423$ & $<0.493=x_{5}+x_{19}$ & $\mathrm{C}<\mathrm{A}+\mathrm{B}$ & $\uparrow$ & $x_{16} \cap x_{17}=0.017$ & $>0.013=x_{16}+x_{17}$ & $\mathrm{C}>\mathrm{A}+\mathrm{B}$ \\
\hline$x_{6} \cap x_{7}=0.169$ & $<0.217=x_{6}+x_{7}$ & $\mathrm{C}<\mathrm{A}+\mathrm{B}$ & $\uparrow$ & $x_{16} \cap x_{18}=0.151$ & $<0.152=x_{16}+x_{18}$ & $\mathrm{C}<\mathrm{A}+\mathrm{B}$ \\
\hline$x_{6} \cap x_{8}=0.552$ & $<0.665=x_{6}+x_{8}$ & $\mathrm{C}<\mathrm{A}+\mathrm{B}$ & $\uparrow$ & $x_{16} \cap x_{19}=0.398$ & $>0.397=x_{16}+x_{19}$ & $\mathrm{C}>\mathrm{A}+\mathrm{B}$ \\
\hline$x_{6} \cap x_{9}=0.228$ & $<0.277=x_{6}+x_{9}$ & $\mathrm{C}<\mathrm{A}+\mathrm{B}$ & $\uparrow$ & $x_{17} \cap x_{18}=0.153$ & $>0.148=x_{17}+x_{18}$ & $\mathrm{C}>\mathrm{A}+\mathrm{B}$ \\
\hline$x_{6} \cap x_{10}=0.282$ & $<0.297=x_{6}+x_{10}$ & $\mathrm{C}<\mathrm{A}+\mathrm{B}$ & $\uparrow$ & $x_{17} \cap x_{19}=0.398$ & $>0.393=x_{17}+x_{19}$ & $\mathrm{C}>\mathrm{A}+\mathrm{B}$ \\
\hline$x_{6} \cap x_{11}=0.179$ & $>0.160=x_{6}+x_{11}$ & $\mathrm{C}>\mathrm{A}+\mathrm{B}$ & $\uparrow \uparrow$ & $x_{18} \cap x_{19}=0.407$ & $<0.532=x_{18}+x_{19}$ & $\mathrm{C}<\mathrm{A}+\mathrm{B}$ \\
\hline$x_{6} \cap x_{12}=0.244$ & $<0.305=x_{6}+x_{12}$ & $\mathrm{C}<\mathrm{A}+\mathrm{B}$ & $\uparrow$ & & & \\
\hline
\end{tabular}

Note: "C" represents the interaction of two factors, $x_{i} \cap x_{j}$; " $\mathrm{A}+\mathrm{B}$ " represents the addition of two factor $q$ values, $\left(q\left(x_{i}\right)+\mathrm{q}\left(x_{j}\right) ;\right.$; $\uparrow$ ” denotes $x_{i}$ and $x_{j}$ enhance each other; “ $\uparrow$ ” denotes a non-linear enhancement of $x_{i}$ and $x_{j}$.

\section{Discussion}

\subsection{Elevation}


of surface vegetation. It is a major factor affecting the distribution of hydrothermal conditions in mountainous regions, and it has certain complexity. With the increase of elevation, the temperature drops, the solar radiation and wind speed increase, the precipitation and relative humidity increase first and then decrease in local regions, and the soil types show significant differences, forming the changes of environmental gradients. This affects the vertical distribution and diversity of plants and causes the different plant types and growth characteristics at different elevations. Relevant studies also showed that elevation has an important impact on the growth of surface vegetation (Li et al.,2019;Chen et al.,2019;Han et al., 2019; Liu et al.,2019).

On the basis of spatial analysis in GIS and geographical detector analysis, we found that area proportion of NDVI coverage with the increase of the elevation (Tab.7), the trend of NDVI change in study area followed the curve-fitting equation:

$$
y=-0.0374 x^{3}+0.3911 x^{2}+0.9307 x, R^{2}=0.972
$$

It can be seen from the curve-fitting equation that area proportion of NDVI coverage showed increased trend as the elevation increased first, and then decreased with the increase of elevation. The vegetation change showed an increasing trend at the elevation of 3561-3792 $\mathrm{m}$ and the vegetation coverage showed a decreasing trend at the elevation of 3561-3792 m. Significant difference in vegetation coverage within the same elevation range (Tab.7). The proportion of low, medium and low vegetation cover to the study area is the smallest in the elevation range of $762-4626 \mathrm{~m}$, while the proportion of medium and high vegetation cover to the study area is the largest. In the elevation range of $4626-5870 \mathrm{~m}$, the proportion of low, medium, medium and high vegetation coverage in the study area reached the maximum, and the proportion of high vegetation coverage in the study area showed a significant trend of decline, or even no vegetation coverage.

\begin{tabular}{llllllll}
\hline Elevation $/ \mathrm{m}$ & $0<\mathrm{NDVI}<0.2$ & $0.2<\mathrm{NDVI}<0.4$ & $0.4<\mathrm{NDVI}<0.6$ & $0.8<\mathrm{NDVI}<0.8$ & $0.8<\mathrm{NDVI}<1$ Area $/ \mathrm{km}^{2}$ & Proportion $/ \%$ \\
\hline $762-1586$ & 0.31 & 8.75 & 43.01 & 125.08 & 365.81 & 542.96 & 2.20 \\
$1586-2034$ & 0.81 & 29.63 & 104.46 & 223.85 & 651.61 & 1010.36 & 4.08 \\
$2034-2412$ & 0.56 & 5.94 & 53.70 & 266.80 & 1076.43 & 1403.43 & 5.67 \\
$2412-2748$ & 0.81 & 0.38 & 14.25 & 192.85 & 1542.34 & 1750.62 & 7.08 \\
$2748-3050$ & 0.38 & 1.31 & 10.88 & 192.60 & 1937.40 & 2142.56 & 8.66 \\
$3050-3319$ & 0.19 & 0.00 & 3.50 & 170.72 & 2486.13 & 2660.53 & 10.76 \\
\hline
\end{tabular}




\begin{tabular}{llllllll}
\hline $3319-3561$ & 0.50 & 1.94 & 5.44 & 143.59 & 3055.53 & 3207.00 & 12.96 \\
$3561-3792$ & 1.31 & 4.00 & 18.13 & 386.44 & 3136.10 & 3545.98 & 14.34 \\
$3792-4032$ & 5.13 & 23.38 & 95.70 & 1208.21 & 1932.27 & 3264.68 & 13.20 \\
$4032-4297$ & 70.20 & 160.90 & 493.77 & 1544.33 & 398.76 & 2667.97 & 10.79 \\
$4297-4626$ & 430.08 & 564.41 & 582.04 & 303.80 & 6.56 & 1886.89 & 7.63 \\
$4626-5870$ & 551.04 & 82.70 & 18.25 & 1.44 & 0.00 & 653.43 & 2.64 \\
Area / $\mathrm{km}^{2}$ & 1061.32 & 883.34 & 1443.13 & 4759.71 & 16588.94 & & \\
Proportion /\% 4.29 & 3.57 & 5.83 & 19.24 & 67.06 & & \\
\hline
\end{tabular}

600

601

602

603

604

605

606

607

608

609

610

611

612

613

614

615

616

617

618

619

620

621

622

623

Studies have shown that the interactions between elevation and GDP density and slope significantly nonlinear enhanced the effect of elevation on NDVI (e.g. $x_{1} \cap x_{15}=0.638>x_{15}$, $\left.x_{15} \cap x_{17}=0.616>x_{15}\right)($ Tab.6). Analysis shows that the interaction of elevation with population density, land use, distance from county,distance from township,distance from road,distance from river, annual average temperature, accumulated temperature $\left(\geq 10^{\circ} \mathrm{C}\right)$,average annual precipitation,moisture index,aridity index, global radiation, geomorphic type, slope,vegetation type, soil type and other factors, the influence of elevation on NDVI is significantly mutual enhanced(Tab.6), (e.g. $x_{2} \cap x_{15}=0.624>x_{15}, x_{3} \cap x_{15}=0.628>x_{15}, x_{4} \cap x_{15}=0.619>x_{15}, x_{5} \cap x_{15}=0.61>$ $x_{15}, x_{6} \cap x_{15}=0.620>x_{15}, x_{7} \cap x_{15}=0.619>x_{15}, x_{8} \cap x_{15}=0.628>x_{15}, x_{9} \cap x_{15}=0.612>x_{15}, x_{10} \cap x_{15}=$ $0.637>x_{15}, x_{11} \cap x_{15}=0.631>x_{15} 、 x_{12} \cap x_{15}=0.609>x_{15}, x_{13} \cap x_{15}=0.629>x_{15}, x_{14} \cap x_{15}=0.627>x_{15}$, $\left.x_{15} \cap x_{16}=0.614>x_{15}, x_{15} \cap x_{18}=0.612>x_{15}, x_{15} \cap x_{19}=0.623>x_{15}\right)$. This implies that the interaction effects significantly enhanced the impact of elevation on NDVI.

\subsection{Annual average temperature (AAT)}

The change of vegetation cover on the Qinghai-Tibet Plateau was positively correlated with the change of temperature over the same period (Liu et al., 2013; Li et al.,2019), and rising temperatures have led to the greening of vegetation on the Tibetan Plateau (Liu et al., 2019). The significant increase of vegetation coverage in Weihe River basin in typical semi-humid and semi-arid regions was more influenced by temperature and soil humidity than precipitation (Liu et al.,2019). However, Vahagn Muradyan et al. (2019) believe that temperature has a negative effect on vegetation NDVI in arid and semi-arid regions of Armenia, which is positively correlated with meadow vegetation NDVI, while precipitation is positively correlated with vegetation NDVI.

The relationship data of vegetation coverage change with average annual temperature in the 

study area were obtained through the spatial superposition analysis and statistical analysis of vegetation coverage map and average annual temperature (Table 14). On the basis of GIS spatial analysis and geographical detector analysis, we found that as the annual average temperature increased (Tab.8), the trend of change in the vegetation coverage area followed the curve-fitting equation:

$$
y=-0.3366 x^{2}+3.8453 x+1.5708, R^{2}=0.8638
$$

It can be seen from the curve-fitting equation that vegetation coverage generally increases first and then decreases with the increase of average annual temperature. According to Tab.8, The vegetation coverage increases at the range of average annual temperature less than $4.873-6.30^{\circ} \mathrm{C}$, and in the area of average annual temperature greater than $6.30{ }^{\circ} \mathrm{C}$, vegetation coverage decreases. The difference of vegetation cover was significant within the same mean annual temperature range (Tab.8). The proportion of low, medium and low vegetation cover in the study area reached the maximum, and the proportion of medium and high vegetation cover in the study area reached the minimum at the range of annual average temperature $<-0.24^{\circ} \mathrm{C}$. In the range of annual average temperature $>2.34{ }^{\circ} \mathrm{C}$, the proportion of low, medium and low covered areas in the study area decreased significantly. The proportion of medium and high vegetation coverage in the study area increased significantly at the range of $2.34-12.04{ }^{\circ} \mathrm{C}$. The proportion of high vegetation coverage in the study area showed a decreasing trend at the range of annual average temperature of $12.04{ }^{\circ} \mathrm{C}$.

Tab. 8 NDVI change with the increase of the annual average temperature

\begin{tabular}{llllllll}
\hline $\mathrm{AAT} /{ }^{\circ} \mathrm{C}$ & $0<\mathrm{NDVI}<0.2$ & $0.2<\mathrm{NDVI}<0.4$ & $0.4<\mathrm{NDVI}<0.6$ & $0.8<\mathrm{NDVI}<0.8$ & $0.8<\mathrm{NDVI}<1$ Area $/ \mathrm{km}^{2}$ & Proportion $/ \%$ \\
\hline$<-1.96$ & 407.30 & 109.89 & 67.55 & 58.47 & 24.20 & 667.41 & 2.70 \\
$-1.96--0.24$ & 463.76 & 409.32 & 503.08 & 420.41 & 176.43 & 1972.99 & 7.98 \\
$-0.24-1.11$ & 148.20 & 214.74 & 465.77 & 1241.06 & 765.20 & 2834.97 & 11.46 \\
$1.11-2.34$ & 37.30 & 41.33 & 165.34 & 1161.41 & 1934.67 & 3340.06 & 13.50 \\
$2.34-3.55$ & 10.08 & 17.14 & 67.55 & 671.44 & 2846.06 & 3612.27 & 14.60 \\
$3.55-4.83$ & 1.01 & 9.07 & 33.27 & 312.53 & 2874.29 & 3230.17 & 13.06 \\
$4.83-6.30$ & 2.02 & 8.07 & 24.20 & 266.16 & 2350.05 & 2650.48 & 10.71 \\
$6.30-7.95$ & 0 & 3.02 & 23.19 & 228.85 & 1965.93 & 2221 & 8.98 \\
$7.95-9.86$ & 1.01 & 2.02 & 21.17 & 207.68 & 1521.33 & 1753.21 & 7.09 \\
$9.86-12.04$ & 0 & 12.10 & 58.47 & 248.01 & 951.71 & 1270.29 & 5.14 \\
$12.04-14.66$ & 2.02 & 21.17 & 83.68 & 177.44 & 617.00 & 901.30 & 3.64 \\
$14.66-18.46$ & 0 & 1.01 & 7.06 & 61.50 & 212.73 & 282.29 & 1.14 \\
\hline
\end{tabular}



shows a non-linear enhancement (Tab.6),i.e, $x_{1} \cap x_{8}=0.567>x_{8}$; the annual average temperature has a mutually enhancement relationship with impact factors, i.e, soil type $\left(x_{8} \cap x_{19}=0.585>x_{8}\right)$, average annual precipitation $\left(x_{8} \cap x_{10}=0.573>x_{8}\right)$, land use $\left(x_{3} \cap x_{8}=0.567>x_{8}\right)$, geomorphic type $\left(x_{8} \cap x_{14}=0.566>x_{8}\right)$, moisture index $\left(x_{8} \cap x_{11}=0.562>x_{8}\right)$, population density $\left(x_{2} \cap x_{8}\right.$ $\left.=0.557>x_{8}\right)$, global radiation $\left(x_{8} \cap x_{13}=0.555>x_{8}\right)$, distance from town $\left(x_{5} \cap x_{8}=0.553>x_{8}\right)$, distance from county seat $\left(x_{4} \cap x_{8}=0.552>x_{8}\right)$, distance from road $\left(x_{6} \cap x_{8}=0.552>x_{8}\right)$, distance from river $\left(x_{7} \cap x_{8}=0.552>x_{8}\right)$, vegetation type $\left(x_{8} \cap x_{18}=0.548>x_{8}\right)$, accumulated temperature $\left(\geq 10^{\circ} \mathrm{C}\right)\left(x_{8} \cap x_{9}=0.547>x_{8}\right)$, slope $\left(x_{8} \cap x_{16}=0.547>x_{8}\right)$, aspect $\left(x_{8} \cap x_{17}=0.546>x_{8}\right)$, aridity index $\left(x_{8} \cap x_{12}=0.543>x_{8}\right)($ Tab.6).

\subsection{Soil type}

Vegetation cover of different soil types is significantly different through spatial overlay analysis and statistical analysis in GIS (Tab.9). According to Tab.9, vegetation coverage of black felt soil (BFS), brown soil (BS), dark brown soil (DBS), Felty soils (FS) and brown soil (BS) accounted for $25.12 \%, 18.78 \%, 16.40 \%, 13.48 \%$ and $12.57 \%$ of the study area, respectively, and the vegetation coverage of other soils accounted for $13.66 \%$ of the study area. Low, medium and low, and medium vegetation coverage were concentrated in the regions of cold permafrost and grassy soil; medium and high vegetation coverage were concentrated in the regions of grassy soil, black felt soil and brown soil; high vegetation coverage was concentrated in the regions of brown soil, grassy felt soil, dark brown soil and brown soil.

According to Tab.6, there is a strong correlation between vegetation types and soil nutrients in the upper reaches of the Minjiang River. The nutrient-water differences between different soil types have a relatively large impact on vegetation growth. The interaction effect significantly enhanced the effect of soil on NDVI. Studies have shown that the soil type can interact with average annual temperature, population density, moisture index, GDP density, slope to significantly nonlinear enhance its influence on NDVI (Tab.6), e.g. $x_{8} \cap x_{19}=0.585>x_{19}, x_{2} \cap x_{19}=$ $0.427>x_{19}, x_{11} \cap x_{19}=0.426>x_{19}, x_{1} \cap x_{19}=0.419>x_{19}, x_{16} \cap x_{19}=0.398>x_{19}, x_{17} \cap x_{19}=0.398>$ $x_{19}$. The interaction of soil type with elevation, geomorphic type,average annual precipitation,land use, distance from river,vegetation type,and distance from county seat on NDVI is significantly 

$\left.x_{3} \cap x_{19}=0.444>x_{19}, x_{7} \cap x_{19}=0.411>x_{19}, x_{18} \cap x_{19}=0.407>x_{19}, x_{4} \cap x_{19}=0.406>x_{19}\right)(\mathrm{Tab} .6)$.

676 Compared with soil, the slope gradient and aspect have weaker effects on vegetation. The possible 677 explanation is that the change of slope gradient and aspect leads to the changes in hydrothermal 678 conditions.

679

680 Tab.9 NDVI change with the increase of the geomorphic types

\begin{tabular}{|c|c|c|c|c|c|c|c|}
\hline Soil types & $0<\mathrm{NDVI}<0.2$ & $0.2<\mathrm{NDVI}<0.4$ & $0.4<\mathrm{NDVI}<0.6$ & $0.8<\mathrm{NDVI}<0.8$ & $0.8<\mathrm{NDVI}<1$ & Area $/ \mathrm{km}^{2}$ & Proportion $/ \%$ \\
\hline BCFS & 0 & 0 & 0 & 1.01 & 48.35 & 49.36 & 0.20 \\
\hline YBS & 1.01 & 0.00 & 0.50 & 45.08 & 534.94 & 581.53 & 2.35 \\
\hline YCS & 0 & 0 & 0 & 1.51 & 161.19 & 162.7 & 0.66 \\
\hline BS & 4.03 & 1.76 & 17.38 & 263.44 & 4358.30 & 4644.91 & 18.78 \\
\hline DBS & 3.78 & 25.94 & 67.24 & 405.73 & 3552.88 & 4055.58 & 16.40 \\
\hline $\mathrm{CS}$ & 38.03 & 91.67 & 267.97 & 794.09 & 1917.60 & 3109.37 & 12.57 \\
\hline GCS & 0 & 0 & 0 & 0.50 & 60.45 & 60.95 & 0.25 \\
\hline LS & 0 & 0 & 0.76 & 14.36 & 288.62 & 303.73 & 1.23 \\
\hline VAS & 0 & 0 & 3.27 & 6.30 & 15.11 & 24.68 & 0.10 \\
\hline RS & 11.58 & 2.01 & 1.26 & 1.01 & 0.50 & 16.37 & 0.07 \\
\hline SS & 1.01 & 0.00 & 5.03 & 58.15 & 539.49 & 603.69 & 2.44 \\
\hline MS & 0 & 0 & 0 & 4.03 & 24.18 & 28.21 & 0.11 \\
\hline BGS & 0 & 0.25 & 3.27 & 34.00 & 51.12 & 88.65 & 0.36 \\
\hline DS & & 0 & 0 & 0 & 1.01 & 1.01 & 0.00 \\
\hline FS & 264.70 & 353.35 & 537.96 & 1335.57 & 842.45 & 3334.02 & 13.48 \\
\hline DFS & 54.65 & 110.31 & 283.33 & 1582.14 & 4183.52 & 6213.95 & 25.12 \\
\hline FFS & 588.07 & 284.84 & 244.55 & 179.32 & 48.10 & 1344.89 & 5.44 \\
\hline YS & 0 & 0 & 1.01 & 17.13 & 30.48 & 48.61 & 0.20 \\
\hline Rock & 59.44 & 4.03 & 0.50 & 0.00 & 0.00 & 63.97 & 0.26 \\
\hline Area $/ \mathrm{km}^{2}$ & 1026.30 & 874.18 & 1434.04 & 4743.36 & 16658.30 & & \\
\hline Proportion $/ \%$ & 4.15 & 3.53 & 5.80 & 19.18 & 67.34 & & \\
\hline
\end{tabular}

681 Note: BCFS,Brown coniferous forest soils; YBS,yellow brown soil; YCS,yellow clunamon soil, BS, brown soil;

682 DBS, dark brown soil; CS,cinnamon soil; GCS, gray cinnamonic soil; LS,limestone soil; VAS, volcanic ash soil;

683 RS,rocky soil; SS,skeleton soil; MS,meadow soil; BGS,boggy soil; DS,Desert solonchaks; FS, Felty soils; DFS,

684 Dark felty soils; FFS, Frigid frozen soils; YS,yellow soil. 

and alpine valleys. Vegetation coverage change with geomorphic types were obtained based on spatial overlay analysis and statistical analysis (Tab.10). According to Tab.10, Vegetation coverage varies significantly with geomorphic types. Vegetation coverage mainly distributed in large undulating mountains and large undulating mountains, accounting for the largest proportion of the study area, $40.86 \%$ and $39.55 \%$, respectively (Tab.10). The vegetation coverage of plains, terraces, hills and extremely undulating mountains accounted for $19.59 \%$ of the study area. The vegetation coverage of different grades varies significantly with the change of geomorphic types. High vegetation coverage is mainly distributed in small undulating mountains, medium undulating mountains and large undulating mountains, accounting for the largest proportion of $65.29 \%$ of the study area. The medium and high vegetation coverage mainly distributed in the medium and large undulating mountains, accounting for the largest proportion of the study area, accounting for 13.82\%. Low, medium and high vegetation coverage mainly distributed in large undulating mountains and extremely undulating mountains, accounting for the largest proportion of $11.63 \%$ of the study area.

Studies have shown that the interaction of geomorphic types with global radiation,GDP density,population density,distance from the county,slope and aspect is significantly nonlinear enhanced (Tab.6), e.g. $x_{13} \cap x_{14}=0.313>x_{14}, x_{1} \cap x_{14}=0.268>x_{19}, x_{2} \cap x_{14}=0.257>x_{14}, x_{4} \cap x_{14}=$ $0.230>x_{14}, x_{14} \cap x_{16}=0.211>x_{14}, x_{14} \cap x_{17}=0.204>x_{14}$. Geomorphic types can interact with elevation,annual average temperature,soil type,land use,aridity index, vegetation type,accumulated temperature $\left(\geq 10^{\circ} \mathrm{C}\right)$, annual average precipitation,distance from township, distance from road, and distance from river to significantly mutual enhance its influence on NDVI (Tab.6), e.g. $x_{14} \cap x_{15}=0.627>x_{14}, x_{8} \cap x_{14}=0.566>x_{14}, x_{14} \cap x_{19}=0.489>x_{14}, x_{3} \cap x_{14} 0.333>x_{14}, x_{12} \cap x_{14}=$ $0.304>x_{14}, x_{14} \cap x_{18}=0.299>x_{14}, x_{9} \cap x_{14}=0.297>x_{14}, x_{10} \cap x_{14}=0.275>x_{14}, x_{5} \cap x_{14}=0.262>$ $x_{14}, x_{6} \cap x_{14}=0.261>x_{14}, x_{7} \cap x_{14} 0.241>x_{14}$.

\begin{tabular}{llllllll}
714 & Tab.10 NDVI change with the increase of the geomorphic types \\
\hline Geomorphic & $0<\mathrm{NDVI}<0.2$ & $0.2<\mathrm{NDVI}<0.4$ & $0.4<\mathrm{NDVI}<0.6$ & $0.8<\mathrm{NDVI}<0.8$ & $0.8<\mathrm{NDVI}<1$ Area $/ \mathrm{km}^{2}$ & Proportion $/ \%$ \\
\hline plain & 1.01 & 1.01 & 6.05 & 31.27 & 66.58 & 105.92 & 0.43 \\
platform & 0.00 & 1.01 & 5.04 & 35.31 & 62.54 & 103.90 & 0.42 \\
hills & 1.01 & 2.02 & 4.04 & 61.54 & 81.71 & 150.31 & 0.61 \\
SUM & 0.00 & 40.35 & 205.79 & 719.27 & 1392.12 & 2357.53 & 9.53 \\
\hline
\end{tabular}




\begin{tabular}{llllllll}
\hline MUM & 5.04 & 31.27 & 124.08 & 1583.80 & 8362.84 & 10107.03 & 40.86 \\
HUM & 448.91 & 447.90 & 655.71 & 1835.99 & 6395.70 & 9784.21 & 39.55 \\
EUM & 575.01 & 355.09 & 393.43 & 547.77 & 256.23 & 2127.53 & 8.60 \\
合计 & 1030.98 & 878.65 & 1394.14 & 4814.94 & 16617.73 & 24736.44 & \\
\hline
\end{tabular}

715 Note: SUM,Small undulating mountain;MUM,Medium undulating mountain; HUM,high undulating mountain;

EUM,Extremely undulating mountain.

717

\subsection{Average annual precipitation (AAP)}

Vegetation coverage increased first and then decreased with the increase of annual mean precipitation, and increased when the annual mean precipitation was less than $651.7 \mathrm{~mm}$ to 939.1 $\mathrm{mm}$, based on spatial superposition analysis and statistical analysis (Tab.11).

According to Tab.11, the area with annual average precipitation $>939.1 \mathrm{~mm}$ or more, the vegetation cover showed a decreasing trend. When the mean annual precipitation was less than $1023.0 \mathrm{~mm}$, the high vegetation cover showed a significant trend with the increase of mean annual precipitation. There were significant differences in vegetation cover within the same average annual precipitation range (Tab.11). The proportion of low, medium and low vegetation coverage in the study area was the smallest, and the proportion of medium and high vegetation coverage in the study area was the largest in the range of annual average precipitation $<1102.0 \mathrm{~mm}$. The area covered by vegetation of different grades showed a decreasing trend within the range of annual average precipitation $>1102.0 \mathrm{~mm}$ (Tab.11).

731

732

Tab.11 NDVI change with the increase of the average annual precipitation

\begin{tabular}{llllllll}
\hline AAP $/ \mathrm{mm}$ & $0<\mathrm{NDVI}<0.2$ & $0.2<\mathrm{NDVI}<0.4$ & $0.4<\mathrm{NDVI}<0.6$ & $0.8<\mathrm{NDVI}<0.8$ & $0.8<\mathrm{NDVI}<1$ Area $/ \mathrm{km}^{2}$ & Proportion $/ \%$ \\
\hline $651.7-715.0$ & 2.016 & 2.016 & 21.171 & 395.187 & 582.700 & 1003.09 & 4.06 \\
$715.0-749.3$ & 19.154 & 17.138 & 34.276 & 374.017 & 833.724 & 1278.31 & 5.17 \\
$749.3-784.0$ & 81.658 & 57.463 & 72.585 & 461.723 & 1045.430 & 1718.86 & 6.95 \\
$784.0-817.3$ & 41.333 & 52.423 & 186.504 & 572.617 & 1112.974 & 1965.85 & 7.95 \\
$817.3-853.5$ & 47.382 & 65.528 & 112.910 & 629.072 & 2135.217 & 2990.11 & 12.09 \\
$853.5-896.3$ & 49.398 & 53.431 & 107.870 & 393.170 & 2932.642 & 3536.51 & 14.30 \\
$896.3-939.1$ & 55.447 & 53.431 & 112.910 & 381.072 & 2983.050 & 3585.91 & 14.50 \\
$939.1-980.3$ & 32.260 & 81.658 & 165.333 & 588.747 & 2256.191 & 3124.19 & 12.63 \\
$980.3-1023.0$ & 98.797 & 122.992 & 276.227 & 513.138 & 1365.007 & 2376.16 & 9.61 \\
$1023.0-1063.2$ & 270.179 & 194.569 & 264.130 & 452.650 & 688.552 & 1870.08 & 7.56 \\
$1063.2-1102.0$ & 260.098 & 117.951 & 114.927 & 222.797 & 227.837 & 943.61 & 3.81 \\
$1102.0-1161.3$ & 115.934 & 30.244 & 51.414 & 70.569 & 75.609 & 343.77 & 1.39
\end{tabular}


Average annual precipitation can interact with global radiation,land use,distance from township, distance from county, population density, GDP density, and other impact factors to significantly nonlinear enhancement on NDVI (Tab.6), e.g. $x_{10} \cap x_{13}=0.326>x_{10}, x_{3} \cap x_{10}=$ $0.295>x_{10}, x_{5} \cap x_{10}=0.277>x_{10}, x_{4} \cap x_{10}=0.264>x_{10}, x_{2} \cap x_{10}=0.260>x_{10}, x_{1} \cap x_{10}=0.249>x_{10}$, $x_{10} \cap x_{16}=0.191>x_{10}, x_{10} \cap x_{17}=0.180>x_{10}$. Average annual precipitation can interact with elevation,annual average temperature, soil type, moisture index,distance from road,aridity index,vegetation type,geomorphic type,accumulated temperature $\left(\geq 10^{\circ} \mathrm{C}\right)$, distance from river and other impact factors to significantly mutually enhancement on NDVI (Tab.6), e.g. $x_{10} \cap x_{15}=$ $0.637>x_{10}, x_{8} \cap x_{10}=0.573>x_{10}, x_{10} \cap x_{19}=0.467>x_{10}, x_{10} \cap x_{11}=0.369>x_{10}, x_{6} \cap x_{10}=0.282>x_{10}$, $x_{10} \cap x_{12}=0.282>x_{10, x_{10}} \cap x_{18}=0.278>x_{10}, x_{10} \cap x_{14}=0.275>x_{10}, x_{9} \cap x_{10}=0.267>x_{10}, x_{7} \cap x_{10}=$ $0.247>x_{10}$

This is consistent with Liu et al. (2013) that NDVI changes in the southeast of the Qinghai-Tibet Plateau are negatively correlated with precipitation changes. Chu et al. (2019) believed that temperature is the dominant factor affecting the growth of vegetation in the Amur-Heilong Jiang basin in spring. NDVI was negatively correlated with precipitation in autumn. This is because precipitation is related to the number of hours of sunlight during photosynthesis, the response of vegetation growth to climate change varies according to vegetation types. The NDVI of coniferous forest, broad-leaved forest, mixed forest and woodland is positively correlated with the temperature of each season, and significantly negatively correlated with autumn precipitation. Therefore, the interaction between annual precipitation and various factors significantly enhanced the effect of dryness index on NDVI.

755

\section{Conclusions}

We quantified individual and interactive influences of impact factors on NDVI changes, and identified the most suitable characteristics of each impact factor for stimulating vegetation growth in the upper reaches of the Minjiang River in Sichuan, western China based on Geographical Detector model. This is a new spatial statistical method to study the interactive effects of impact factors on the spatial patterns of vegetation cover distribution and the vegetation changes using remotely sensed data and spatial analysis in GIS. It is both timely and necessary to understand the NDVI changes and its driving forces, to provide theoretical guidance and decision-making basis 
for constructing ecological barrier and sustainable management in the upper reaches of the Minjiang River or to mitigate environmental degradation in other regions of China experiencing rapid vegetation changes. We have extracted the vegetation changes in the upper reaches of the Minjiang River, Sichuan, estern China from 2000 to 2015 from RS and GIS.

We found that the temporal and spatial changes of vegetation cover is significant. In 2000 and 2015, the spatial variation of vegetation cover in the upper reaches of the Minjiang River was significantly different. The high vegetation cover (NDVI>0.8) areas were mainly distributed in the upper reaches of the Minjiang River at an elevation below $3600 \mathrm{~m}$. The low vegetation cover $(\mathrm{NDVI}<0.2)$ areas were mainly distributed in the extremely high-elevation regions of the western and north-eastern upper reaches of the Minjiang River. The areas of mid-high and high vegetation cover accounted for $17 \%, 68 \%$ and $20 \%$ and $65 \%$ or higher of the upper reaches of the Minjiang River, respectively. The changes of vegetation cover from 2000 to 2015 were mainly in the regions of the upper reaches of the Minjiang River and its tributaries and were characterized by the significant transformation of the areas of mid-high vegetation cover (NDVI $>0.6)$ or above. The roll-out areas of $0.6<\mathrm{NDVI}<0.8$ and NDVI $>0.8$ were $1680 \mathrm{~km}^{2}$ and $2080 \mathrm{~km}^{2}$, and the roll-in areas of them were $2415 \mathrm{~km}^{2}$ and $1185 \mathrm{~km}^{2}$, respectively, leading to the increase and decrease of the $0.6<\mathrm{NDVI}<0.8$ and NDVI $>0.8$ areas, respectively. And this resulted in the increase and decrease of the areas of mid-high vegetation cover $(0.6<\mathrm{NDVI}<0.8)$ and high vegetation cover (NDVI>0.8), respectively.

We illustrated the individual influences of impact factors on NDVI changes. Impact factors can be ranked in descending order by the magnitude of their influence on NDVI: elevation > annual average temperature $>$ soil type $>$ geomorphic type $>$ dryness index $>$ annual precipitation $>$ land use type $>$ accumulated temperature $\left(\geq 10^{\circ} \mathrm{C}\right)>$ vegetation type $>$ distance from road $>$ distance from township $>$ distance from river $>$ total radiation $>$ humidity index $>$ population density $>$ distance from county $>$ GDP density $>$ slope gradient $>$ slope aspect. Firstly, the elevation, annual average temperature and soil type all had an explanatory power above $39 \%$, which were the main factors affecting vegetation change and well explained the changes of vegetation cover. Secondly, the explanatory power of the geomorphic type, dryness index, annual precipitation, land use type, vegetation type, accumulated temperature $\left(\geq 10^{\circ} \mathrm{C}\right)$ and distance from road was all above $11 \%$. 
We found that interactive effects of impact factors on NDVI changes, and the synergistic effect of impact factors is manifested as mutual enhancement and nonlinear enhancement. This study reveals the suitable characteristics of the main factors that promote vegetation growth, helps the better understanding of the effects of impact factors on NDVI. This is a critical step discovering the driving mechanism of NDVI change. The findings in this study may, to some extent, help to intervene and promote vegetation change by determining a favourable value range or the most suitable characteristics of impact factors, so as to promote ecological protection and vegetation restoration and alleviate environmental degradation.

Finally, this study highlights the advantages of the Geographical Detector model in detecting NDVI spatial differentiations and revealing their driving factors. The traditional principal component analysis, classical regression models, etc., are usually based on some assumptions or constraints, such as the normal distribution and linear hypothesis to analyse the relationship between vegetation NDVI and its driving factors. Compared with the previous methods, Geographic detector is a new statistical method to detect spatial differentiation and reveal the driving factors behind it. This method has no linear hypothesis, elegant form and clear physical meaning. It uses the spatial heterogeneity to detect the consistency of spatial distribution patterns between the dependent variables and the independent variables, and based on this, measures the explanation power of independent variables on dependent variables. It is better in detecting the explanatory factors and analyse the interactive relationships between variables than the conventional statistics.

\section{Acknowledgments}

Funding for this study was provided by Applied Basic Research Project of Sichuan Province, China(No.2017JY0155),Humanities and Social Science Research Foundation of Ministry of Education, China (No.17YJA850007) and National Natural Science Foundation of China (No.41371125; No.32060370). The authors thank the editors and anonymous referees for their valuable comments and suggestions, which helped improve the manuscript. Landsat data was acquired from the USGS EROS Data Center and Data Center for Resources and Environmental Sciences, Chinese Academy of Sciences (RESDC) (http://www.resdc.cn). The funding sources had no involvement in the collection, analysis and interpretation of data; the writing of the report; and 
the decision to submit the article for publication.

\section{References}

Anvar Sanaei.,Mingshi Li.,Arshad Ali.(2019). Topography, grazing, and soil textures control over rangelands' vegetation quantity and quality. Science of The Total Environment 697:134-153.

Chen, C. N., Zhu L Q.,Tian L.,Li X G. (2019). Spatial-temporal changes in vegetation characteristics and climate in the Qinling-Daba Mountains. Acta Ecologica Sinica 39(9): 3257-3266.

C.Piedallu.,V.Chéret.,J.P.Denux.,V.Perez.,J.S.Azcona.,I.Seynave.,J.C.Gégout.(2019). Soil and climate differently impact NDVI patterns according to the season and the stand type. Science of the Total Environment 651(Part 2):2874-2885.

Chu,H.H.,Sergey Venevsky.,Wu, C.,Wang, MH..(2019). NDVI-based vegetation dynamics and its response to climate changes at Amur-Heilongjiang River Basin from 1982 to 2015. Science of The Total Environment 650(Part 2):2051-2062.

Han,J.C.,Huang,Y.F.,Zhang,H.,Wu, X.F.(2019). Characterization of elevation and land cover dependent trends of NDVI variations in the Hexi region, northwest China. Journal of Environmental Management 232:1037-1048.

Ke,Y.H.,Jungho Im.,Lee,J.H.,Gong,H.L.,Youngryel Ryu.(2016). Characteristics of Landsat 8 OLI-derived NDVI by comparison with multiple satellite sensors and in-situ observations. Remote Sensing of Environment 164:298-313.

Li,L.H.,Zhang,Y.L.,Wu,J.S.,Li,S.C.,Zhang,B.H.,Zu,J.X.,Zhang,H.M.,Ding,M.J.,Basanta Paudel.(2019). Increasing sensitivity of alpine grasslands to climate variability along an elevational gradient on the Qinghai-Tibet Plateau. Science of The Total Environment 678:21-29.

Liu, J.H.,Gao, J.X.,Wang, W.J. (2013).Variations of Vegetation Coverage and Its Relations to Global Climate Changes on the Tibetan Plateau during 1981-2005. Journal of Mountain Science 31(2):234-242.

Liu,L.B.,Wang,Y.,Wang,Z.,Li,D.L.,Zhang,Y.T.,Qin, D.H.,Li,S.C.(2019). Elevation-dependent decline in vegetation greening rate driven by increasing dryness based on three satellite NDVI datasets on the Tibetan Plateau. Ecological Indicators,doi.org/10.1016/j.ecolind.2019 .105569

Liu, S.Y.,Huang,S.Z.,Xie,Y.Y.,Wang,H.,Huang,Q.,Leng, G.Y.,Li Pei.,Wang, L. (2019a). Spatial-temporal changes in vegetation cover in a typical semi-humid and semi-arid region in China: Changing patterns, causes and implications. Ecological Indicators 98:462-475. 
Luke Wallace.,Daisy S.Saldias.,Karin Reinke.,Samuel Hillman.,BryanHally.,Simon Jones.(2019). Using orthoimages generated from oblique terrestrial photography to estimate and monitor vegetation cover. Ecological Indicators 101:91-101.

Ma,H.X., Cheng, C.C.,Song, Y.Q., Ye,S.,Hu,Y.M.(2018).The dynamic change of grassland vegetation cover and its driving factors in Qinghai Province in recent 10 years.Research of Soil and Water Conservation 25(6):137-145.

Ma,Q.F.,Zhu,L.P.,Lü, X.M.,Wang,J.B.,Ju,J.T.,Thomas Kasper.,Gerhard Daut.,Torsten Haberzettl.(2019). Late glacial and Holocene vegetation and climate variations at Lake Tangra Yumco, central Tibetan Plateau.Global and Planetary Change 174:16-25.

Mo, K.L., Chen, Q.W.,Chen, C.,Zhang, J.Y.,Wang, L.,Bao, Z.X.(2019). Spatiotemporal variation of correlation between vegetation cover and precipitation in an arid mountain-oasis river basin in northwest China. Journal of Hydrology 574:138-147.

Peng, W.F.,Kuang, T.T.,Tao, S.(2019). Quantifying influences of natural factors on vegetation NDVI changes based on geographical detector in Sichuan, western China. Journal of Cleaner Production 233:353-367.

Peng, W.F., Wang, G.J., Zhou, J.M., Xu, X.L., Luo, H.L., Zhao, J.F., Yang, C.J.(2016). Dynamic monitoring of fractional vegetation cover along Minjiang River from Wenchuan County to Dujiangyan City using multi-temporal Landsat 5 and 8images. Acta Ecologica Sinica 36 (7):1975-1988.

Sun,W.C.,Wang,Y.Y.,Fu, Y.S.,Xue, B.L.,Wang, G.Q.,Yu,J.S.,Zuo,D.P.,Xu, Z.X.(2019). Spatial heterogeneity of changes in vegetation growth and their driving forces based on satellite observations of the Yarlung Zangbo River Basin in the Tibetan Plateau. Journal of Hydrology 574: 324-332.

Yao,R.,Wang,L.C.,Huang, X.,Chen, X.X.,Liu, Z.J.(2019). Increased spatial heterogeneity in vegetation greenness due to vegetation greening in mainland China.Ecological Indicators 99:240-250.

Vahagn Muradyan.,Garegin Tepanosyan.,Shushanik Asmaryan., Armen Saghatelyan.,Fabio Dell'Acqu.(2019). Relationships between NDVI and climatic factors in mountain ecosystems: A case study of Armenia. Remote Sensing Applications: Society and Environment 14:158-169.

Xiong,Y.L.,Zhang,K.L.,Zhu,Y.H.,Li,X.(2018).Multi-Scale Coupling Relationships between NDVI and environmental factors in sub-watershed of the Minjiang River upper reaches. Journal of Sichuan Agricultural University 36(6):792-797.

Wang, J.F., Li, X.H., Christakos G, et al. (2010). Geographical detectors-based health risk assessment and its application in the neural tube defects study of the Heshun region, China. International Journal of 
884 Wang, J.F., Zhang, T.L., Fu, B.J. (2016). A measure of spatial stratified heterogeneity. Ecological Indicators 67:

885

886

887

888

889

890

891

892

893

894

895 250-256.

Wang, J.F, Xu, C.D. (2017). Geodetector: Principle and prospective. Acta Geographica Sinica 72(1): 116-134.

Yang, R.R.,Yi, G.H.,Zang, T.B.,Li, J.J.,Bie, X.J.,Xia, J.,Sheng, Y.L. (2018). Analysis of vegetation cover change and climate factors driving in Zoige region from 2000 to 2015. Pratacultural Science 35(12):2822-2835.

Zhang, S.Y., Zhang,Y.,Wang, C.Q.,Zeng, Z.C.Vegetation coverage and its correlation with topographic factors in the upper reaches of Minjiang River basin.(2018). Water and Soil Conservation Bulletin 38(1):69-75.

Zhu, C.,Peng, W.F.,Zhang, L.F.,Luo, Y.,Dong,Y.B.,Wang, M.F.(2019). Study of temporal and spatial variation and driving force of fractional vegetation cover in upper reaches of Minjiang River from 2006 to 2016. Acta Ecologica Sinica 39(5):1583-1594. 


\section{Supplementary Files}

This is a list of supplementary files associated with this preprint. Click to download.

- Highlights.doc 